\title{
Information Geometric Perspective on Off-Resonance Effects in Driven Two-Level Quantum Systems
}

\author{
Carlo Cafaro ${ }^{1, *}$, Steven Gassner ${ }^{1}$ and Paul M. Alsing ${ }^{2}$ \\ 1 SUNY Polytechnic Institute, Albany, NY 12203, USA; SGassner@sunypoly.edu \\ 2 Air Force Research Laboratory, Information Directorate, Rome, NY 13441, USA; paul.alsing@us.af.mil \\ * Correspondence: carlocafaro2000@yahoo.it
}

Received: 16 January 2020; Accepted: 26 January 2020; Published: 5 February 2020

\begin{abstract}
We present an information geometric analysis of off-resonance effects on classes of exactly solvable generalized semi-classical Rabi systems. Specifically, we consider population transfer performed by four distinct off-resonant driving schemes specified by $\mathrm{su}(2 ; \mathbb{C})$ time-dependent Hamiltonian models. For each scheme, we study the consequences of a departure from the on-resonance condition in terms of both geodesic paths and geodesic speeds on the corresponding manifold of transition probability vectors. In particular, we analyze the robustness of each driving scheme against off-resonance effects. Moreover, we report on a possible tradeoff between speed and robustness in the driving schemes being investigated. Finally, we discuss the emergence of a different relative ranking in terms of performance among the various driving schemes when transitioning from on-resonant to off-resonant scenarios.
\end{abstract}

Keywords: information geometry; quantum systems; resonance

\section{Introduction}

It is well-known that the resonance phenomenon concerns the amplification that occurs when the frequency of an externally applied field (for instance, a magnetic field) is equal to a natural characteristic frequency of the system on which it acts. When a field is applied at the resonant frequency of another system, the system oscillates at a higher amplitude than when the field is applied at a non-resonant frequency. In an actual experimental laboratory, off-resonance effects can occur for a number of reasons. For instance, a common source of off-resonance effects is the presence of unforeseen magnetic field gradients that are not part of the externally applied magnetic field. These gradients can cause signal loss and, as a consequence, signals at the "wrong" resonant frequency (that is, off-resonance). Furthermore, when the intensity of an applied magnetic field gradient is changed in a laboratory, decaying eddy currents can produce time-varying off-resonant effects [1]. Additionally, the presence of concomitant gradients can create off-resonance effects in the form of $x y$-spatial variations in the Larmor frequency when a magnetic field gradient in the $z$-direction is applied [2]. For the interested reader, we discuss two illustrative examples concerning the departure from the on-resonance condition in the context of classical and quantum mechanics in Appendix A.

The resonance phenomenon happens to play a key role in problems where one seeks to drive an initial source state towards a final target state. For instance, typical problems of this kind can be found when controlling population transfer in quantum systems [3-5] or when searching for a target state [6,7]. In the framework of quantum control theory, it is commonly believed that an off-resonant driving scheme cannot help achieving population transfer with high fidelity between two quantum states. However, there do exist theoretical investigations relying on the phenomenon of superoscillations [8] where population transfer occurs by using only off-resonant driving fields [9]. In particular, during resonant superoscillations, the quantum system is temporarily excited and behaves 
as if truly driven at resonance [9]. Furthermore, the existence of suboptimal transfer efficiencies, caused by the sequential use of two lasers in order to physically realize a transition from highly excited Rydberg states to ground states [10,11], leads to question the possibility of transition from a ground state to the Rydberg state by means of lasers far-off-resonant with respect to the transition frequency. Indeed, motivated by such possibility, driving schemes specified by periodic square-wells and Gaussian pulses in order to implement coherent transfer with far-off-resonant driving fields have been proposed in reference [12].

It is also well-known that on-resonance driving is not a good control strategy for a quantum system if, from an experimental standpoint, one allows the Rabi frequency (that is, the frequency that describes the coupling between the driving field and the two-level system) to be higher than the Larmor frequency (that is, the characteristic transition frequency of the two-level system in the absence of the external driving field) [13]. This is called the strong driving regime. In such a scenario, the so-called rotating wave approximation (RWA, [14]) is not applicable and the system is driven with an external field whose amplitude is greater than or equal to the energy splitting between the system's states. For an analysis on the deviation from sinusoidal Rabi oscillations by studying the time-evolution dynamics of a strongly driven dressed electron spin in silicon, we refer to reference [15]. Furthermore, for an explicit manifestation of strong sensitivity to the initial phase of the driving field in the dynamics of a general semi-classical Rabi model in regimes of arbitrary strong driving, we refer to reference [16].

Based upon our considerations, it is clear that off-resonance phenomena exhibit both experimental (for instance, limited control of spurious electromagnetic signals and strong driving regimes) and theoretical (for instance, sensitivity to initial conditions and emergence of more complex dynamical scenarios) interest. In the framework of analog quantum searching, we have recently presented a detailed investigation concerning the physical connection between quantum search Hamiltonians and exactly solvable time-dependent two-level quantum systems in reference [7]. In references [17,18], instead, we analyzed the possibility of modifying the original Farhi-Gutmann Hamiltonian quantum search algorithm [19] in order to speed up the procedure for producing a suitably distributed unknown normalized quantum mechanical state provided only a nearly optimal fidelity is sought. In reference [20], we presented an information geometric characterization of the oscillatory or monotonic behavior of statistically parametrized squared probability amplitudes originating from special functional forms of the Fisher information function: constant, exponential decay, and power-law decay. Furthermore, for each case, we computed both the computational speed and the availability loss of the corresponding physical processes by exploiting a convenient Riemannian geometrization of useful thermodynamical concepts. Finally, building upon our works presented in references $[7,17,18,20]$, we presented in reference [21] an information geometric analysis of geodesic speeds and entropy production rates in geodesic motion on manifolds of parametrized quantum states. These pure states emerged as outputs of suitable su $(2 ; \mathbb{C})$ time-dependent Hamiltonian evolutions used to describe distinct types of analog quantum search schemes viewed as driving strategies. In particular, by evaluating the geodesic speed and the total entropy production along the optimum transfer paths in a number of physical scenarios of interest in analog quantum search problems (for instance, constant, oscillatory, power-law decay, and exponential decay of the driving magnetic fields), we showed in an explicit quantitative manner that to a faster transfer there corresponds necessarily a higher entropy production rate. Thus, we concluded that lower entropic efficiency values do appear to accompany higher entropic geodesic speed values in quantum transfer processes. Our information geometric analysis in reference [21] was limited to the case in which the on-resonance driving condition was satisfied for all quantum driving strategies. However, based upon our previous considerations, it would be of theoretical interest to investigate from an information geometric perspective the effects of off-resonance effects on these driving strategies. In particular, we would like to address the following questions: 
(i) What are the main effects of deviations from the on-resonance condition in these su $(2 ; \mathbb{C})$ time-dependent Hamiltonian evolutions?

(ii) Do off-resonance effects modify (with respect to the on-resonance scenario) the relative ranking in terms of performance (quantified in terms of geodesic speed and/or minimum transfer time) among the driving schemes being considered?

(iii) Are there driving schemes that are especially robust against deviations from the on-resonance condition and that, in addition, are capable of reaching sufficiently high fidelity values?

The rest of the paper is organized as follows. In Section 2, we present our driving strategies in terms of classes of exactly solvable generalized semi-classical Rabi systems. Specifically, we consider the population transfer performed by four distinct off-resonant driving schemes specified by su $(2 ; \mathbb{C})$ time-dependent Hamiltonian models. In Section 3, we propose our information geometric analysis of off-resonance effects on such classes of systems. For each scheme, we study the consequences of a departure from the on-resonance condition in terms of both geodesic paths and geodesic speeds on the corresponding manifold of transition probability vectors. In particular, we analyze the robustness of each driving scheme against off-resonance effects. Moreover, we report on a possible tradeoff between speed and robustness in the driving schemes being investigated. Finally, we discuss the emergence of a different relative ranking in terms of performance among the various driving schemes when transitioning from the on-resonant to the off-resonant scenarios. Our conclusive remarks appear in Section 4. Auxiliary illustrative examples together with some technical details are placed in Appendices A and B.

\section{The su $(2 ; \mathbb{C})$ Quantum Driving}

In this section, we introduce our driving strategies in terms of classes of exactly solvable generalized semi-classical Rabi systems [22,23].

The quantum evolution we take into consideration is specified by means of an Hamiltonian operator $\mathcal{H}_{\mathrm{su}(2 ; \mathbb{C})}$ written as the most general linear superposition of the three traceless and anti-Hermitian generators $\left\{i \sigma_{x},-i \sigma_{y}, i \sigma_{z}\right\}$ of $\mathrm{su}(2 ; \mathbb{C})$, the Lie algebra of the special unitary group $\mathrm{SU}(2 ; \mathbb{C})$,

$$
\mathcal{H}_{\mathrm{su}(2 ; \mathbb{C})}(t) \stackrel{\text { def }}{=} a(t)\left(i \sigma_{x}\right)+b(t)\left(-i \sigma_{y}\right)+c(t)\left(i \sigma_{z}\right) .
$$

In Equation (1), $a(t), b(t)$, and $c(t)$ are time-dependent complex coefficients while $\vec{\sigma} \stackrel{\text { def }}{=}$ $\left(\sigma_{x}, \sigma_{y}, \sigma_{z}\right)$ denotes the Pauli vector operator. In particular, by putting $a(t) \stackrel{\text { def }}{=}-i \omega_{x}(t), b(t) \stackrel{\text { def }}{=} i \omega_{y}(t)$, and $c(t) \stackrel{\text { def }}{=}-i \Omega(t)$, Equation (1) yields

$$
\mathcal{H}_{\mathrm{su}(2 ; \mathbb{C})}(t) \stackrel{\text { def }}{=} \omega_{x}(t) \sigma_{x}+\omega_{y}(t) \sigma_{y}+\Omega(t) \sigma_{z}
$$

In the framework of su $(2 ; \mathbb{C})$ Hamiltonian models, $\omega(t) \stackrel{\text { def }}{=} \omega_{x}(t)-i \omega_{y}(t)=\omega_{\mathcal{H}}(t) e^{i \phi_{\omega}(t)}$ and $\Omega(t)$ are the so-called complex transverse field and real longitudinal field, respectively. Obviously, $\omega_{\mathcal{H}}(t)$ denotes the modulus of $\omega(t)$. In what follows, we assume that longitudinal fields $\Omega(t)$ are oriented along the $z$-axis while transverse fields $\omega(t)$ lie in the $x y$-plane. Taking into consideration the quantum evolution of an electron (or, more generally, a spin-1/2 particle) in an external time-dependent magnetic field $\vec{B}(t)$, the Hamiltonian $\mathcal{H}_{\mathrm{su}(2 ; \mathbb{C})}(t)$ in Equation (2) can be rewritten as

$$
\mathcal{H}_{\mathrm{su}(2 ; \mathbb{C})}(t) \stackrel{\text { def }}{=}-\vec{\mu} \cdot \vec{B}(t),
$$

where $\vec{\mu} \stackrel{\text { def }}{=}(e \hbar / 2 m c) \vec{\sigma}$ denotes the magnetic moment of the electron with $\mu_{\text {Bohr }} \stackrel{\text { def }}{=}|e| \hbar /(2 m c) \simeq$ $9.27 \times 10^{-21}[\mathrm{cgs}]$ being the so-called Bohr magneton. The quantity $m \simeq 9.11 \times 10^{-31}[\mathrm{MKSA}]$ is the mass of an electron while $|e| \simeq 1.60 \times 10^{-19}[\mathrm{MKSA}]$ denotes the absolute value of the electric charge 
of an electron. Moreover, $c \simeq 3 \times 10^{8}[\mathrm{MKSA}]$ and $\hbar \simeq 1.05 \times 10^{-34}[\mathrm{MKSA}]$ are the speed of light and the reduced Planck constant, respectively. The magnetic field $\vec{B}(t)$ in Equation (1) can be recast as

$$
\vec{B}(t) \stackrel{\text { def }}{=} \vec{B}_{\perp}(t)+\vec{B}_{\|}(t),
$$

with $\vec{B}_{\perp}(t) \stackrel{\text { def }}{=} B_{x}(t) \hat{x}+B_{y}(t) \hat{y}$ and $\vec{B}_{\|}(t) \stackrel{\text { def }}{=} B_{z}(t) \hat{z}$. Equating the Hamiltonians in Equations (2) and (3) and making use of the magnetic field decomposition in Equation (4), the connection between the set of field intensities $\left\{\omega_{\mathcal{H}}(t), \Omega_{\mathcal{H}}(t)\right\}$ and the set of magnetic field intensities $\left\{B_{\perp}(t), B_{\|}(t)\right\}$ becomes clear. More specifically, we observe that $B_{\perp}(t) \propto \omega_{\mathcal{H}}(t)$ and $B_{\|}(t) \propto \Omega_{\mathcal{H}}(t) \stackrel{\text { def }}{=}|\Omega(t)|$. The exact connection between the components $\left\{B_{x}(t), B_{y}(t), B_{z}(t)\right\}$ and $\left\{\omega_{x}(t), \omega_{y}(t), \Omega(t)\right\}$ is expressed by

$$
B_{x}(t)=-\frac{2 m c}{e \hbar} \omega_{x}(t), B_{y}(t)=-\frac{2 m c}{e \hbar} \omega_{y}(t) \text {, and } B_{z}(t)=-\frac{2 m c}{e \hbar} \Omega(t) .
$$

Moreover, in terms of field intensities, we get

$$
B_{\perp}(t)=\frac{2 m c}{|e| \hbar} \omega_{\mathcal{H}}(t) \text {, and } B_{\|}(t)=\frac{2 m c}{|e| \hbar} \Omega_{\mathcal{H}}(t) .
$$

Studying the quantum evolution of an electron governed by the Hamiltonian in Equation (3) by means of exact analytical expressions of complex probability amplitudes and/or real transition probabilities from an initial source state to a final target state can be quite challenging. In particular, the canonical matrix representations of $\mathcal{H}_{\mathrm{su}(2 ; \mathbb{C})}$ in Equation (2) and $\mathcal{U}(t)$, the unitary evolution operator arising from $\mathcal{H}_{\mathrm{su}(2 ; \mathbb{C})}$, with $i \hbar \dot{\mathcal{U}}(t)=\mathcal{H}_{\mathrm{su}(2 ; \mathbb{C})} \mathcal{U}(t)$ and $\dot{\mathcal{U}} \stackrel{\text { def }}{=} \partial_{t} \mathcal{U}$, are given by

$$
\left[\mathcal{H}_{\mathrm{su}(2 ; \mathbb{C})}\right] \stackrel{\text { def }}{=}\left(\begin{array}{cc}
\Omega(t) & \omega(t) \\
\omega^{*}(t) & -\Omega(t)
\end{array}\right) \text {, and }[\mathcal{U}(t)] \stackrel{\text { def }}{=}\left(\begin{array}{cc}
\alpha(t) & \beta(t) \\
-\beta^{*}(t) & \alpha^{*}(t)
\end{array}\right),
$$

respectively. The unitarity of the quantum evolution demands that the complex probability amplitudes $\alpha(t)$ and $\beta(t)$ fulfill the normalization condition, $|\alpha(t)|^{2}+|\beta(t)|^{2}=1$. Once the unitary evolution operator $\mathcal{U}(t)$ in Equation (7) is given, the temporal evolution of a quantum source state $|s\rangle$,

$$
|s\rangle \stackrel{\text { def }}{=} x|w\rangle+\sqrt{1-x^{2}}\left|w_{\perp}\right\rangle,
$$

can be characterized by the following transformation law,

$$
\left(\begin{array}{c}
x \\
\sqrt{1-x^{2}}
\end{array}\right) \rightarrow\left(\begin{array}{c}
\alpha(t) x+\beta(t) \sqrt{1-x^{2}} \\
-\beta^{*}(t) x+\alpha^{*}(t) \sqrt{1-x^{2}}
\end{array}\right)
$$

with $x \stackrel{\text { def }}{=}\langle w \mid s\rangle$ denoting the quantum overlap. The set of orthonormal state vectors $\left\{|w\rangle,\left|w_{\perp}\right\rangle\right\}$ generate the two-dimensional search space of the $N=2^{n}$-dimensional complex Hilbert space $\mathcal{H}_{2}^{n}$. Therefore, using Equations (7), (8), and (9), the probability that the source state $|s\rangle$ transitions into the target state $|w\rangle$ under $\mathcal{U}(t)$ becomes

$$
\mathcal{P}_{|s\rangle \rightarrow|w\rangle}(t) \stackrel{\text { def }}{=}|\langle w|\mathcal{U}(t)| s\rangle|^{2}=|\alpha(t)|^{2} x^{2}+|\beta(t)|^{2}\left(1-x^{2}\right)+\left[\alpha(t) \beta^{*}(t)+\alpha^{*}(t) \beta(t)\right] x \sqrt{1-x^{2}} .
$$

It is evident from Equation (10) that in order to calculate the exact analytical expression of transition probabilities, one must have the exact analytical formula of the evolution operator $\mathcal{U}(t)$ expressed in terms of the complex probability amplitudes $\alpha(t)$ and $\beta(t)$. Ideally, having specified the fields $\omega(t)$ and $\Omega(t)$ by means of physical arguments, one would solve the coupled system of 
first order ordinary differential equations with time-dependent coefficients emerging from the relation $i \hbar \dot{\mathcal{U}}(t)=\mathcal{H}_{\mathrm{su}(2 ; \mathbb{C})} \mathcal{U}(t)$ with $\mathcal{U}(0)=\mathcal{I}$,

$$
i \hbar \dot{\alpha}(t)=\Omega(t) \alpha(t)-\omega(t) \beta^{*}(t) \text {, and } i \hbar \dot{\beta}(t)=\omega(t) \alpha^{*}(t)+\Omega(t) \beta(t),
$$

where $\alpha(0)=1$ and $\beta(0)=0$. However, it is often the case that this general approach does not yield exact analytical solutions. It is recognized that it is rather difficult to find exact analytical solutions in time-dependent two-level quantum systems specified by equations as the ones in Equation (11). A very powerful technique for investigating these types of quantum evolutions is the so-called rotating coordinates technique. This method, originally proposed by Rabi, Ramsey, and Schwinger in magnetic resonance problems, can be presented as a three-step technique: First, recast the original problem from a stationary to a rotating frame of reference by performing a suitable change of coordinates; second, find the solution to the simplified problem viewed in a rotating frame of reference by means of rotating coordinates; third, solve the original problem by an inverse transformation from the rotating to the stationary frame. Usually, the rotating frame of reference rotates about the axis specified by the magnetic field $\vec{B}_{\|}$with the angular frequency characterized by the magnetic field $\vec{B}_{\perp}$. In quantum terms, assume that in a static frame of reference the quantum evolution of the qubit is ruled by the Schrödinger equation,

$$
i \hbar \partial_{t}|\psi(t)\rangle=\mathcal{H}(t)|\psi(t)\rangle .
$$

Then, considering a unitary transformation $T$ between two distinct vector bases $\{|\psi(t)\rangle\}$ and $\left\{\left|\psi^{\prime}(t)\right\rangle\right\}$, the Schrödinger equation in the new frame of reference can be written as

$$
i \hbar \partial_{t}\left|\psi^{\prime}(t)\right\rangle=\mathcal{H}^{\prime}(t)\left|\psi^{\prime}(t)\right\rangle,
$$

with $\left|\psi^{\prime}(t)\right\rangle \stackrel{\text { def }}{=} T|\psi(t)\rangle$ and $\mathcal{H}^{\prime}(t) \stackrel{\text { def }}{=}\left[T \mathcal{H}(t) T^{\dagger}+i \hbar\left(\partial_{t} T\right) T^{\dagger}\right]$. More specifically, assuming that the unitary transformation $T \stackrel{\text { def }}{=} e^{-i \sigma_{z} \frac{\phi_{\omega}(t)}{2}}$ is a rotation by an angle $\phi_{\omega}(t)$ about the $z$-axis, the Hamiltonians in Equations (2) and (3) can be recast as

$$
\mathcal{H}_{\mathrm{su}(2 ; \mathbb{C})}^{\prime}(t) \stackrel{\text { def }}{=}\left[\Omega(t)+\frac{\hbar}{2} \dot{\phi}_{\omega}(t)\right] \sigma_{z}+\omega_{\mathcal{H}}(t) \sigma_{x}
$$

and,

$$
\mathcal{H}_{\mathrm{su}(2 ; \mathbb{C})}^{\prime}(t) \stackrel{\text { def }}{=}\left[-\frac{e \hbar}{2 m c} B_{\|}(t)+\frac{\hbar}{2} \dot{\phi}_{\omega}(t)\right] \sigma_{z}+\frac{e \hbar}{2 m c} B_{\perp}(t) \sigma_{x}
$$

respectively, where $\dot{\phi}_{\omega} \stackrel{\text { def }}{=} d \phi_{\omega} / d t$. In the so-called original Rabi scenario, $\phi_{\omega}(t) \stackrel{\text { def }}{=} \omega_{0} t$ with the angular frequency $\omega_{0}$ being a negative real constant while the magnetic field intensities $B_{\|}$and $B_{\perp}$ are constant. In particular, assuming the so-called static resonance condition,

$$
\omega_{0}=\frac{e}{m c} B_{\|}
$$

the Hamiltonian in Equation (15) specifies a time-independent quantum mechanical problem. Unlike the original Rabi scenario, in the so-called generalized Rabi scenario proposed by Messina and collaborators in references [22,23], $B_{\|}, B_{\perp}$, and $\phi_{\omega}$ are arbitrary time-dependent dynamical variables. In particular, assuming the so-called generalized Rabi condition,

$$
\dot{\phi}_{\omega}(t)=-\frac{2}{\hbar} \Omega(t),
$$

the Hamiltonians in Equations (14) and (15) do not yield a time-independent quantum mechanical problem. Inspired by our research reported in reference [7] and exploiting the findings in references [22,23], we take into consideration here four quantum mechanical scenarios where the 
transition probability $\mathcal{P}_{\left|w_{\perp}\right\rangle \rightarrow|w\rangle}(t)$ from an initial state $\left|w_{\perp}\right\rangle$ to a final state $|w\rangle$, with $\left\langle w_{\perp} \mid w\right\rangle=\delta_{w_{\perp}, w}$, $\sigma_{z}|w\rangle=+|w\rangle$, and $\sigma_{z}\left|w_{\perp}\right\rangle=-\left|w_{\perp}\right\rangle$, can be analytically described. In all four cases, we assume to be in a physical scenario in which,

$$
\dot{\phi}_{\omega}(t)=\omega_{0} \text {, and } \Omega(t)=-\frac{\hbar}{2} \omega_{0},
$$

with $\omega_{0}$ being a negative real constant. We point out that, from a pure mathematical viewpoint, more general temporal behaviors of $\dot{\phi}_{\omega}(t)$ and $\Omega(t)$ in Equation (18) could have been selected provided Equation (17) is fulfilled. However, the choice adopted in Equation (18) seems to be more suitable from an experimental standpoint. The four scenarios can be formally distinguished by means of the temporal behavior of the intensity $\omega_{\mathcal{H}}(t)$ of the complex transverse field $\omega(t)$. In the first case, we assume a constant field intensity $\omega_{\mathcal{H}}(t)$,

$$
\omega_{\mathcal{H}}^{(1)}(t) \stackrel{\text { def }}{=} \Gamma .
$$

This first case specifies the original Rabi scenario where $\mathcal{P}_{\left|w_{\perp}\right\rangle \rightarrow|w\rangle}(t)$ is given by,

$$
\mathcal{P}_{\left|w_{\perp}\right\rangle \rightarrow|w\rangle}^{(1)}(t)=\sin ^{2}\left(\frac{\Gamma}{\hbar} t\right)
$$

In the remaining three cases, we take into consideration three generalized Rabi scenarios where the field intensity $\omega_{\mathcal{H}}(t)$ manifests oscillatory, power law decay, and exponential law decay behaviors,

$$
\omega_{\mathcal{H}}^{(2)}(t) \stackrel{\text { def }}{=} \Gamma \cos (\lambda t), \omega_{\mathcal{H}}^{(3)}(t) \stackrel{\text { def }}{=} \frac{\Gamma}{(1+\lambda t)^{2}} \text {, and } \omega_{\mathcal{H}}^{(4)}(t) \stackrel{\text { def }}{=} \Gamma e^{-\lambda t},
$$

respectively. Note that $\omega_{\mathcal{H}}^{(2)}(t)$ in Equation (21) is a positive quantity on a temporal scale with $0 \leq t \leq(\pi / 2) \lambda^{-1}$. In all three cases, it can be proven that the transition probability $\mathcal{P}_{\left|w_{\perp}\right\rangle \rightarrow|w\rangle}^{(j)}(t)$ is given by [23],

$$
\mathcal{P}_{\left|w_{\perp}\right\rangle \rightarrow|w\rangle}^{(j)}(t)=\sin ^{2}\left[\int_{0}^{t} \frac{\omega_{\mathcal{H}}^{(j)}\left(t^{\prime}\right)}{\hbar} d t^{\prime}\right],
$$

for any $j \in\{2,3,4\}$. Interestingly, being on resonance, the transition probabilities In Equations (20) and (22) for all four cases depend only on the integral of the transverse field intensity $\omega_{\mathcal{H}}(t)$.

Before beginning our information geometric analysis, we formally introduce here an adimensional parameter $\beta_{0}$ that quantifies the departure from the on-resonance condition,

$$
\beta_{0} \stackrel{\text { def }}{=} \frac{\hbar}{2 \omega_{\mathcal{H}}(t)}\left[\dot{\phi}_{\omega}(t)+\frac{2}{\hbar} \Omega(t)\right] .
$$

When $\beta_{0}$ assumes a nonzero constant value, the generalized expression of the transition probability in Equation (22) is given by [23],

$$
\mathcal{P}_{\left|w_{\perp}\right\rangle \rightarrow|w\rangle}^{(j)}\left(t ; \beta_{0}\right) \stackrel{\text { def }}{=} \frac{1}{1+\beta_{0}^{2}} \sin ^{2}\left[\sqrt{1+\beta_{0}^{2}} \int_{0}^{t} \frac{\omega_{\mathcal{H}}^{(j)}\left(t^{\prime}\right)}{\hbar} d t^{\prime}\right] .
$$

We emphasize that $\beta_{0}$ in Equation (23) is generally a time-dependent quantity. However, in what follows, we shall limit our information geometric analysis to the case in which $\beta_{0}$ is a constant parameter with transition probabilities given in Equation (24). For further details on the link between $\beta_{0}$ in Equation (23) and its analogue counterpart in the framework of Rabi's original static off-resonance condition, we refer to Appendix A. 


\section{Information Geometric Analysis}

In this section, we present our information geometric analysis of off-resonance effects on the chosen quantum driving strategies. For each scheme, we analyze the consequences of a departure from the on-resonance condition in terms of both geodesic paths and geodesic speeds on the corresponding manifold of transition probability vectors.

\subsection{Preliminaries}

In order to provide some conceptual background for our forthcoming information geometric analysis that emerges from quantum driving strategies, we begin by introducing some preliminary remarks.

Assume to consider an $N \stackrel{\text { def }}{=} 2^{n}$-dimensional complex Hilbert space $\mathcal{H}_{2}^{n}$ together with two neighboring normalized pure states $|\psi(\theta)\rangle$ and $\left|\psi^{\prime}(\theta)\right\rangle$ where,

$$
|\psi\rangle \stackrel{\text { def }}{=} \sum_{l=1}^{N} \sqrt{p_{l}} e^{i \varphi_{l}}|l\rangle \text { and }\left|\psi^{\prime}\right\rangle \stackrel{\text { def }}{=} \sum_{l=1}^{N} \sqrt{p_{l}+d p_{l}} e^{i\left(\varphi_{l}+d \varphi_{l}\right)}|l\rangle,
$$

respectively. In Equation (25), $\{|l\rangle\}$ with $1 \leq l \leq N$ denotes an orthonormal basis of $\mathcal{H}_{2}^{n}$ while $p_{l}=p_{l}(\theta)$ and $\varphi_{l}=\varphi_{l}(\theta)$ are real functions of a continuous real parameter $\theta$. The distinguishability metric on this manifold of Hilbert space rays is specified by the Fubini-Study metric [24],

$$
d s_{\mathrm{FS}}^{2} \stackrel{\text { def }}{=}\left\{\cos ^{-1}\left[\left|\left\langle\psi^{\prime} \mid \psi\right\rangle\right|\right]\right\}^{2}=g_{a b}^{(\mathrm{FS})}(\theta) d \theta^{a} d \theta^{b},
$$

where the Fubini-Study metric tensor components $g_{a b}^{(\mathrm{FS})}$ are related to the Fisher-Rao metric tensor components $\mathcal{F}_{a b}(\theta)$ by the condition,

$$
g_{a b}^{(\mathrm{FS})}(\theta)=\frac{1}{4}\left[\mathcal{F}_{a b}(\theta)+4 \sigma_{a b}^{2}(\theta)\right] .
$$

The quantities $\mathcal{F}_{a b}(\theta)$ and $\sigma_{a b}^{2}(\theta)$ in Equation (27) are given by,

$$
\mathcal{F}_{a b}(\theta) \stackrel{\text { def }}{=} \sum_{l=1}^{N} \frac{1}{p_{l}(\theta)} \frac{\partial p_{l}(\theta)}{\partial \theta^{a}} \frac{\partial p_{l}(\theta)}{\partial \theta^{b}}
$$

and,

$$
\sigma_{a b}^{2}(\theta) \stackrel{\text { def }}{=} \sum_{l=1}^{N} \frac{\partial \varphi_{l}(\theta)}{\partial \theta^{a}} \frac{\partial \varphi_{l}(\theta)}{\partial \theta^{b}} p_{l}(\theta)-\left(\sum_{k=1}^{N} \frac{\partial \varphi_{l}(\theta)}{\partial \theta^{a}} p_{l}(\theta)\right)\left(\sum_{k=1}^{N} \frac{\partial \varphi_{l}(\theta)}{\partial \theta^{b}} p_{l}(\theta)\right),
$$

respectively. In what follows, we assume that the non-negative term $\sigma_{a b}^{2}(\theta)$ in Equation (29) (which denotes the variance of the phase changes) is equal to zero. This working assumption can be justified by rephasing in a suitable fashion the basis vectors used to write the state $|\psi(\theta)\rangle$. Specifically, the rephasing procedure demands that $\operatorname{Im}\left[\langle\psi(\theta) \mid l\rangle\left\langle l \mid d \psi_{\perp}(\theta)\right\rangle\right]=0$, for any $1 \leq l \leq N$. The state $\left|d \psi_{\perp}\right\rangle \stackrel{\text { def }}{=}|d \psi\rangle-\langle\psi \mid d \psi\rangle|d \psi\rangle$ denotes the projection of $|d \psi\rangle$ orthogonal to $|\psi\rangle$ where $|d \psi\rangle \stackrel{\text { def }}{=}\left|\psi^{\prime}\right\rangle-|\psi\rangle$ while $|\psi\rangle$ and $\left|\psi^{\prime}\right\rangle$ are given in Equation (25). In summary, for a convenient choice of the basis vectors $\{|l\rangle\}$ used for the decomposition in Equation $(25), g_{a b}^{(\mathrm{FS})}(\theta)$ becomes proportional to $\mathcal{F}_{a b}(\theta)$ as evident from Equation (27). We refer to reference [24] for further details. For the sake of completeness, we emphasize that if the basis $\{|l\rangle\}$ satisfies the above mentioned conditions, the basis vectors can always be rephased so that both $\langle l \mid \psi\rangle$ and $\left\langle l \mid d \psi_{\perp}\right\rangle$ are real. These latter conditions, in turn, are reminiscent of the so-called parallel transport conditions that occur in the context of Berry's description of a geometric effect in the shape of an additional phase factor emerging after an adiabatic and cyclic transport of a quantum system [25]. Specifically, in the framework of Berry's phase analysis 
generalized by Aharonov and Anandan by removing the adiabaticity constraint in the cyclic quantum evolution [26], it is possible to show that there exists one distinct curve in the Hilbert space $\mathcal{H}$ fulfilling the parallel transport conditions. Namely, two neighboring states $|\psi(\theta)\rangle$ and $|\psi(\theta+d \theta)\rangle$ in $\mathcal{H}$ have the same phase, that is to say, $\langle\psi(\theta) \mid \psi(\theta+d \theta)\rangle$ is real and positive. For such a curve, the dynamical phase vanishes. In summary, for special Hamiltonians that satisfy a special gauge choice (that is, the parallel transport conditions), the dynamical phase vanishes [27].

In our paper, the parameter $\theta$ is the statistical version of the elapsed time $t$. That is, we assume $\theta$ is a parameter that can be experimentally specified by measurement of a suitable observable that changes with time (for instance, the transverse magnetic field intensity $B_{\perp}(t)$ ). For further details on the concept of statistical elapsed time, we refer to reference [28]. In particular, we suppose that the output $|\psi(\theta)\rangle$ of a quantum driving Hamiltonian acting on the input defined by the normalized source state $|s\rangle \stackrel{\text { def }}{=}\left|\psi\left(\theta_{0}\right)\right\rangle$ can be described as,

$$
\left|\psi\left(\theta_{0}\right)\right\rangle \mapsto|\psi(\theta)\rangle \stackrel{\text { def }}{=} e^{i \varphi_{w}(\theta)} \sqrt{p_{w}(\theta)}|w\rangle+e^{i \varphi_{w_{\perp}}(\theta)} \sqrt{p_{w_{\perp}}(\theta)}\left|w_{\perp}\right\rangle .
$$

In general, the normalized output state $|\psi(\theta)\rangle$ is an element of the two-dimensional subspace of the $n$-qubit complex Hilbert space $\mathcal{H}_{2}^{n}$ accommodating the source state $|s\rangle$ and generated by the set of orthonormal state vectors $\left\{|w\rangle,\left|w_{\perp}\right\rangle\right\}$. The modulus squared of the probability amplitudes $p_{w}(\theta) \stackrel{\text { def }}{=}|\langle w \mid \psi(\theta)\rangle|^{2}$ and $p_{w_{\perp}}(\theta) \stackrel{\text { def }}{=}\left|\left\langle w_{\perp} \mid \psi(\theta)\right\rangle\right|^{2}$ stand for the success and failure probabilities of the given driving strategy, respectively. Furthermore, $\varphi_{w}(\theta)$ and $\varphi_{w_{\perp}}(\theta)$ denote the real quantum phases of the states $|w\rangle$ and $\left|w_{\perp}\right\rangle$, respectively. We note that the quantum state $|\psi(\theta)\rangle$ in Equation (30) is parameterized by means of a single continuous real parameter that emerges from the elapsed time of the driving strategy. As previously mentioned, this parameter $\theta$ mimics a statistical macrovariable used to distinguish neighboring quantum states $|\psi(\theta)\rangle$ and $|\psi(\theta)\rangle+|d \psi(\theta)\rangle$ along a path through the space of pure quantum states. In summary, we focus on the space of probability distributions $\{p(\theta)\}$,

$$
|\psi(\theta)\rangle \mapsto p(\theta) \stackrel{\text { def }}{=}\left(p_{w}(\theta), p_{w_{\perp}}(\theta)\right)=\left(|\langle w \mid \psi(\theta)\rangle|^{2},\left|\left\langle w_{\perp} \mid \psi(\theta)\right\rangle\right|^{2}\right),
$$

where the natural Riemannian distinguishability metric between two neighboring probability distributions $p(\theta)$ and $p(\theta+d \theta)$ is given by the Fisher-Rao information metric in Equation (28). For an overview of the use of information geometric techniques to analog quantum search problems, we refer to references [20,29-31].

In any quantum driving scheme considered in this paper, following references [22,23], we assume the success probability $p_{0}(\theta)$ and the failure probability $p_{1}(\theta)$ can be recast as

$$
p_{0}(\theta) \stackrel{\text { def }}{=} \mathcal{A} \sin ^{2}[\Sigma(\theta)] \text { and, } p_{1}(\theta) \stackrel{\text { def }}{=} 1-\mathcal{A} \sin ^{2}[\Sigma(\theta)],
$$

respectively. In Equation (32), the quantity $\theta$ denotes the temporal parameter while the quantity $\mathcal{A}$ is defined as

$$
\mathcal{A}=\mathcal{A}\left(\beta_{0}\right) \stackrel{\text { def }}{=} \frac{1}{1+\beta_{0}^{2}},
$$

with $\beta_{0}$ being the (assumed) constant parameter that quantifies the deviation from the on-resonance condition. The quantity $\Sigma(\theta)$, instead, specifies the type of driving being considered. The Fisher information $\mathcal{F}(\theta)$ corresponding to the probability vector $\left(p_{0}(\theta), p_{1}(\theta)\right)$ is given by [32,33],

$$
\mathcal{F}(\theta) \stackrel{\text { def }}{=} \frac{\dot{p}_{0}^{2}(\theta)}{p_{0}(\theta)}+\frac{\dot{p}_{1}^{2}(\theta)}{p_{1}(\theta)}
$$


with $\dot{p} \stackrel{\text { def }}{=} d p / d \theta$. Substituting Equation (32) into Equation (34), the Fisher information $\mathcal{F}(\theta)$ becomes

$$
\mathcal{F}(\theta) \stackrel{\text { def }}{=} 4 \mathcal{A}^{2}\left(\frac{d \Sigma}{d \theta}\right)^{2}\left\{\frac{\cos ^{2}[\Sigma(\theta)]}{\mathcal{A}}+\frac{\cos ^{2}[\Sigma(\theta)] \sin ^{2}[\Sigma(\theta)]}{1-\mathcal{A} \sin ^{2}[\Sigma(\theta)]}\right\}
$$

Note that when we are on-resonance, $\beta_{0}=0, \mathcal{A}=1$, and $\mathcal{F}(\theta)$ reduces to

$$
\mathcal{F}(\theta) \stackrel{\text { def }}{=} 4\left(\frac{d \Sigma}{d \theta}\right)^{2}
$$

In particular, when $\Sigma(\theta)$ is linear in $\theta, \mathcal{F}(\theta)=4$ as reported in reference [20]. Finally, once the expression of the Fisher information $\mathcal{F}(\theta)$ is given, the geodesic equation to integrate becomes

$$
\frac{d^{2} \theta}{d \tilde{\zeta}^{2}}+\frac{1}{2 \mathcal{F}(\theta)} \frac{d \mathcal{F}(\theta)}{d \theta}\left(\frac{d \theta}{d \xi}\right)^{2}=0
$$

For more details on how to derive Equation (37) and how to express the quantum mechanical infinitesimal Fubini-Study line element in terms of the Fisher information and/or the Fisher-Rao information metric, we refer to reference [20]. For the sake of completeness, we observe that Equation (37) can be recast as,

$$
\ddot{\theta}+\frac{\dot{\mathcal{F}}}{2 \mathcal{F}} \dot{\theta}=0,
$$

where $\dot{\theta} \stackrel{\text { def }}{=} d \theta / d \xi$ and $\dot{\mathcal{F}} \stackrel{\text { def }}{=} d \mathcal{F} / d \xi$. Defining $w \stackrel{\text { def }}{=} \dot{\theta}$ and after some straightforward algebraic manipulations, Equation (38) reduces to

$$
\frac{d \mathcal{F}}{\mathcal{F}}=-2 \frac{d w}{w}
$$

Integration of Equation (39) yields a closed-form implicit relation between the dependent variable $\theta$ and the independent variable $\xi$,

$$
c(\theta, \xi)=\int_{\theta\left(\xi_{0}\right)}^{\theta(\xi)} \sqrt{\mathcal{F}(\theta)} d \theta-\int_{\xi_{0}}^{\xi} a_{0} d \xi=0,
$$

with $a_{0}$ being a real constant of integration. Equation (40) is an implicit constraint equation between the variables $\theta$ and $\xi$. Despite the closed-form implicit relation in Equation (40), in what follows we shall numerically find the geodesic paths satisfying Equation (37) for the four driving strategies defined in Equations (19) and (21).

\subsection{Geodesic Paths}

In what follows, after specifying $\Sigma(\theta)$ in Equation (32) and $\mathcal{F}(\theta)$ in Equation (35), we shall focus on numerically finding from Equation (37) the geodesic paths $\theta=\theta(\xi)$ that correspond to the various off-resonant driving schemes.

\subsubsection{Constant Behavior}

In the first case, we assume $\Sigma(\theta)$ is defined as

$$
\Sigma(\theta) \stackrel{\text { def }}{=} \mathcal{B} \theta .
$$

Therefore, from Equations (32) and (41), the probabilities $p_{0}(\theta)$ and $p_{1}(\theta)$ are given by

$$
p_{0}(\theta) \stackrel{\text { def }}{=} \mathcal{A} \sin ^{2}[\mathcal{B} \theta] \text { and, } p_{1}(\theta) \stackrel{\text { def }}{=} 1-\mathcal{A} \sin ^{2}[\mathcal{B} \theta],
$$


respectively. In Equation (42), the quantity $\mathcal{A}$ is defined in Equation (33) while $\mathcal{B}$ is given by

$$
\mathcal{B}=\mathcal{B}\left(\beta_{0}, \Gamma, \hbar\right) \stackrel{\text { def }}{=} \sqrt{1+\beta_{0}^{2}} \frac{\Gamma}{\hbar},
$$

respectively. In Figure 1, we plot the success probability $p_{0}(\theta)$ versus the temporal parameter $\theta$ in the case of constant driving with the assumption that $\beta_{0} \in\{0,1 / 4,1 / 2,1\}$. From Figure 1 , we note that the maximum value of the success probability decreases with respect to increasing values of the parameter $\beta_{0}$. Furthermore, the periodic oscillatory behavior of the success probability is also affected by nonzero values of $\beta_{0}$. In particular, to higher values of $\beta_{0}$ there correspond smaller (higher) values of the period (frequency) of oscillation. In this first case, putting Equation (42) into Equation (35), the Fisher information becomes

$$
\mathcal{F}_{\text {constant }}^{\text {(off-resonance) }}(\theta) \stackrel{\text { def }}{=} 4 \mathcal{A B}^{2}\left\{\frac{1+\cos (2 \mathcal{B} \theta)}{2-\mathcal{A}[1-\cos (2 \mathcal{B} \theta)]}\right\} .
$$

For the sake of completeness, note that when $\beta_{0}=0, \mathcal{A}=1$, and $\mathcal{F}(\theta)$ in Equation (44) reduces to

$$
\mathcal{F}_{\text {constant }}^{\text {(on-resonce) }}(\theta)=4 \mathcal{B}^{2} \text {. }
$$

Finally, employing Equations (37) and (44), the geodesic equation to integrate becomes

$$
\frac{d^{2} \theta}{d \xi^{2}}-\frac{(1-\mathcal{A}) \mathcal{B} \tan (\mathcal{B} \theta)}{1-\mathcal{A} \sin ^{2}(\mathcal{B} \theta)}\left(\frac{d \theta}{d \xi}\right)^{2}=0
$$

The output arising from the numerical integration of Equation (46) is plotted in Figure 2.

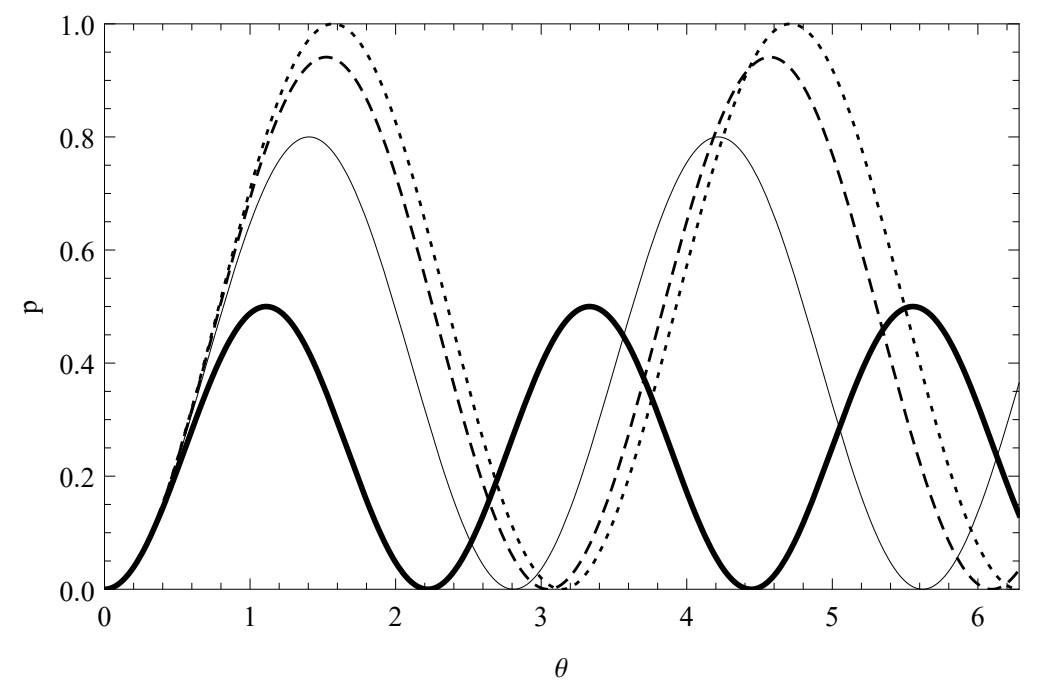

Figure 1. Plot of the success probability $p$ versus the temporal parameter $\theta$ in the case of constant driving for $\beta=0$ (dotted), $\beta_{0}=0.25$ (dashed), $\beta_{0}=0.5$ (thin solid), and $\beta_{0}=1$ (thick solid).

\subsubsection{Oscillatory Behavior}

In this second scenario, we assume $\Sigma(\theta)$ is given by

$$
\Sigma(\theta) \stackrel{\text { def }}{=} \frac{\mathcal{B}}{\lambda} \sin (\lambda \theta) .
$$

Therefore, from Equations (32) and (47), the probabilities $p_{0}(\theta)$ and $p_{1}(\theta)$ become

$$
p_{0}(\theta) \stackrel{\text { def }}{=} \mathcal{A} \sin ^{2}\left[\frac{\mathcal{B}}{\lambda} \sin (\lambda \theta)\right] \text { and, } p_{1}(\theta) \stackrel{\text { def }}{=} 1-\mathcal{A} \sin ^{2}\left[\frac{\mathcal{B}}{\lambda} \sin (\lambda \theta)\right],
$$


respectively. In this case, employing Equation (47), the Fisher information in Equation (35) becomes

$$
\mathcal{F}_{\text {oscillatory }}^{\text {(off-resonance) }}(\theta) \stackrel{\text { def }}{=} \frac{\left\{\frac{d\left(\mathcal{A} \sin ^{2}\left[\frac{\mathcal{B}}{\lambda} \sin (\lambda \theta)\right]\right)}{d \theta}\right\}^{2}}{\mathcal{A} \sin ^{2}\left[\frac{\mathcal{B}}{\lambda} \sin (\lambda \theta)\right]}+\frac{\left\{\frac{d\left(1-\mathcal{A} \sin ^{2}\left[\frac{\mathcal{B}}{\lambda} \sin (\lambda \theta)\right]\right)}{d x}\right\}^{2}}{1-\mathcal{A} \sin ^{2}\left[\frac{\mathcal{B}}{\lambda} \sin (\lambda \theta)\right]},
$$

that is, after some straightforward but tedious algebra,

$$
\mathcal{F}_{\text {oscillatory }}^{\text {(off-renance) }}(\theta)=8 \mathcal{A B}^{2} \cos ^{2}(\lambda \theta) \frac{\cos ^{2}\left[\frac{\mathcal{B}}{\lambda} \sin (\lambda \theta)\right]}{2-\mathcal{A}\left\{1-\cos \left[2 \frac{\mathcal{B}}{\lambda} \sin (\lambda \theta)\right]\right\}} .
$$

Observe that when $\beta_{0}=0, \mathcal{A}=1$, and, recalling that $1+\cos (2 x)=2 \cos ^{2}(x), \mathcal{F}(\theta)$ in Equation (50) reduces to

$$
\mathcal{F}_{\text {oscillatory }}^{\text {(on-resonce) }}(\theta)=4 \mathcal{B}^{2} \cos ^{2}(\lambda \theta) .
$$

Finally, employing Equations (37) and (50), the geodesic equation to integrate in this second scenario can be formally written as

$$
\frac{d^{2} \theta}{d \xi^{2}}+\frac{1}{2 \mathcal{F}_{\mathrm{o}}(\theta)} \frac{d \mathcal{F}_{\mathrm{o}}(\theta)}{d \theta}\left(\frac{d \theta}{d \xi}\right)^{2}=0
$$

with $\mathcal{F}_{\mathrm{O}}(\theta) \stackrel{\text { def }}{=} \mathcal{F}_{\text {oscillatory }}^{\text {(off-res) }}(\theta)$ as in Equation (50). Using a symbolic mathematical software (Mathematica, for instance) together with the analytical knowledge of what happens in the limiting case of $\beta_{0}=0$ [21], Equation (52) can be finally recast as

$$
\frac{d^{2} \theta}{d \xi^{2}}-\left\{\lambda \tan (\lambda \theta)+\frac{(1-\mathcal{A}) \mathcal{B} \cos (\lambda \theta) \tan \left[\frac{\mathcal{B}}{\lambda} \sin (\lambda \theta)\right]}{1-\mathcal{A} \sin ^{2}\left[\frac{\mathcal{B}}{\lambda} \sin (\lambda \theta)\right]}\right\}\left(\frac{d \theta}{d \bar{\zeta}^{\xi}}\right)^{2}=0 .
$$

The output emerging from numerically integrating Equation (53) is plotted in Figure 2.
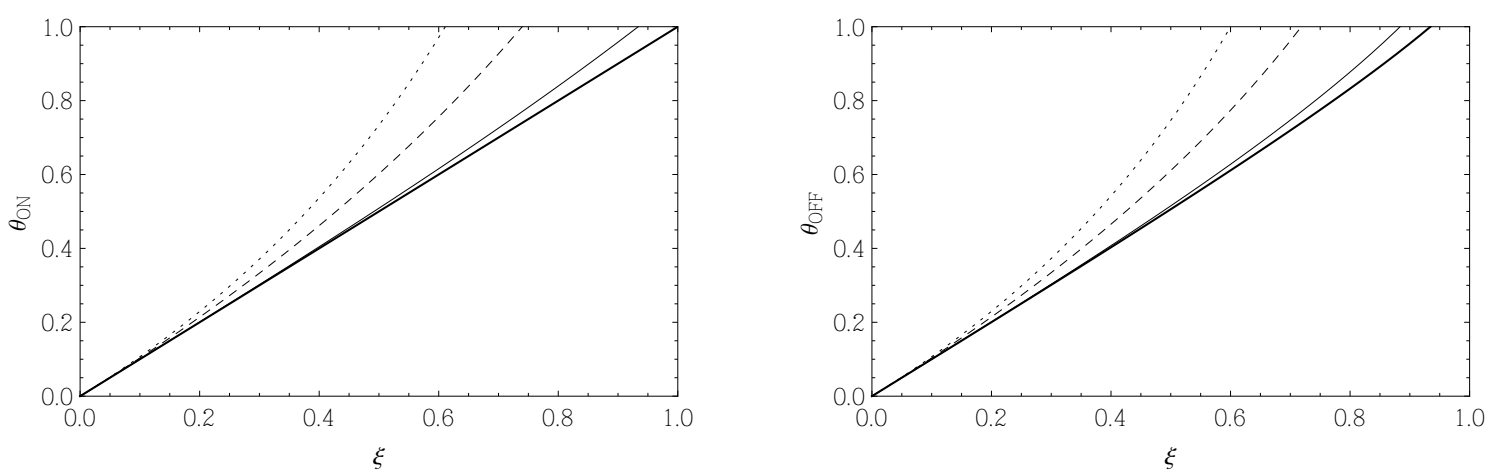

Figure 2. Numerical plots of the geodesic paths $\theta$ versus the affine parameter $\xi$ for the four driving strategies: Power-law (dotted line), exponential (dashed line), oscillatory (thin solid line), and constant (thick solid line). In the LHS (left-hand-side), we consider the on-resonance scenario with $\beta_{0}=0$. In the RHS (right-hand-side), we consider the off-resonance scenario with $\beta_{0}=1 / 2$. In both sides, we set $\Gamma / \hbar=1, \lambda=2 / \pi, \theta_{0}=0$, and $\dot{\theta}_{0}=1$.

\subsubsection{Power Law Decay}

In this third scenario, we assume $\Sigma(\theta)$ is defined as

$$
\Sigma(\theta) \stackrel{\text { def }}{=} \frac{\mathcal{B}}{\lambda}\left(1-\frac{1}{1+\lambda \theta}\right) .
$$


Therefore, the probabilities $p_{0}(\theta)$ and $p_{1}(\theta)$ in Equation (32) can be rewritten as

$$
p_{0}(\theta) \stackrel{\text { def }}{=} \mathcal{A} \sin ^{2}\left[\frac{\mathcal{B}}{\lambda}\left(1-\frac{1}{1+\lambda \theta}\right)\right] \text { and, } p_{1}(\theta) \stackrel{\text { def }}{=} 1-\mathcal{A} \sin ^{2}\left[\frac{\mathcal{B}}{\lambda}\left(1-\frac{1}{1+\lambda \theta}\right)\right],
$$

respectively. In this case, the Fisher information in Equation (35) can be formally recast as

$$
\mathcal{F}_{\text {power-law-decay }}^{\text {(off-resonance) }}(\theta) \stackrel{\text { def }}{=} \frac{\left\{\frac{d\left(\mathcal{A} \sin ^{2}\left[\frac{\mathcal{B}}{\lambda}\left(1-\frac{1}{1+\lambda \theta}\right)\right]\right)}{d \theta}\right\}^{2}}{\mathcal{A} \sin ^{2}\left[\frac{\mathcal{B}}{\lambda}\left(1-\frac{1}{1+\lambda \theta}\right)\right]}+\frac{\left\{\frac{d\left(1-\mathcal{A} \sin ^{2}\left[\frac{\mathcal{B}}{\lambda}\left(1-\frac{1}{1+\lambda \theta}\right)\right]\right)}{d x}\right\}^{2}}{1-\mathcal{A} \sin ^{2}\left[\frac{\mathcal{B}}{\lambda}\left(1-\frac{1}{1+\lambda \theta}\right)\right]^{2}},
$$

that is, after some tedious algebra,

$$
\mathcal{F}_{\text {power-law-decay }}^{\text {(off-resonance) }}(\theta)=\frac{4 \mathcal{A B}^{2}}{(1+\lambda \theta)^{4}} \frac{1+\cos \left(2 \frac{\mathcal{B} \theta}{1+\lambda \theta}\right)}{2-\mathcal{A}\left[1-\cos \left(2 \frac{\mathcal{B} \theta}{1+\lambda \theta}\right)\right]} .
$$

Once again, for the sake of completeness, we point out that when $\beta_{0}=0, \mathcal{A}=1$, and $\mathcal{F}(\theta)$ in Equation (57) reduces to

$$
\mathcal{F}_{\text {power-law-decay }}^{\text {(on-resonance) }}(\theta)=\frac{4 \mathcal{B}^{2}}{(1+\lambda \theta)^{4}} .
$$

Finally, making use of Equations (37) and (57), the geodesic equation to integrate in this third scenario can be formally written as

$$
\frac{d^{2} \theta}{d \xi^{2}}+\frac{1}{2 \mathcal{F}_{\text {pld }}(\theta)} \frac{d \mathcal{F}_{\text {pld }}(\theta)}{d \theta}\left(\frac{d \theta}{d \xi}\right)^{2}=0,
$$

with $\mathcal{F}_{\text {pld }}(\theta) \stackrel{\text { def }}{=} \mathcal{F}_{\text {power-law-decay }}^{\text {(off-renace) }}(\theta)$ as in Equation (57). Employing a symbolic mathematical software (Mathematica, for instance) together with the analytical knowledge of what occurs in the on-resonance case where $\beta_{0}=0$ [21], Equation (59) can be finally recast as

$$
\frac{d^{2} \theta}{d \xi^{2}}-\left\{\frac{2 \lambda}{1+\lambda \theta}+\frac{(1-\mathcal{A}) \mathcal{B} \tan \left(\frac{\mathcal{B} \theta}{1+\lambda \theta}\right)}{(1+\lambda \theta)^{2}\left[1-\mathcal{A} \sin ^{2}\left(\frac{\mathcal{B} \theta}{1+\lambda \theta}\right)\right]}\right\}\left(\frac{d \theta}{d \tilde{\zeta}}\right)^{2}=0 .
$$

In Figure 2, we plot the output that arises from the numerical integration of Equation (60).

\subsubsection{Exponential Decay}

In this last scenario, we assume

$$
\Sigma(\theta) \stackrel{\text { def }}{=} \frac{\mathcal{B}}{\lambda}\left(1-e^{-\lambda \theta}\right) .
$$

Therefore, the success and failure probabilities probabilities $p_{0}(\theta)$ and $p_{1}(\theta)$ are given by

$$
p_{0}(\theta) \stackrel{\text { def }}{=} \mathcal{A} \sin ^{2}\left[\frac{\mathcal{B}}{\lambda}\left(1-e^{-\lambda \theta}\right)\right] \text { and, } p_{1}(\theta) \stackrel{\text { def }}{=} 1-\mathcal{A} \sin ^{2}\left[\frac{\mathcal{B}}{\lambda}\left(1-e^{-\lambda \theta}\right)\right],
$$

respectively. In this case, the Fisher information in Equation (35) is formally given by

$$
\mathcal{F}_{\text {exponential-decay }}^{\text {(off-resonanc) }}(\theta) \stackrel{\text { def }}{=} \frac{\left\{\frac{d\left(\mathcal{A} \sin ^{2}\left[\frac{\mathcal{B}}{\lambda}\left(1-e^{-\lambda \theta}\right)\right]\right)}{d \theta}\right\}^{2}}{\mathcal{A} \sin ^{2}\left[\frac{\mathcal{B}}{\lambda}\left(1-e^{-\lambda \theta}\right)\right]}+\frac{\left\{\frac{d\left(1-\mathcal{A} \sin ^{2}\left[\frac{\mathcal{B}}{\lambda}\left(1-e^{-\lambda \theta}\right)\right]\right)}{d x}\right\}^{2}}{1-\mathcal{A} \sin ^{2}\left[\frac{\mathcal{B}}{\lambda}\left(1-e^{-\lambda \theta}\right)\right]^{2}},
$$


that is,

$$
\mathcal{F}_{\text {exponential-decay }}^{\text {(off-resonace }}(\theta) \stackrel{\text { def }}{=} 4 \mathcal{A B}^{2} e^{-2 \lambda \theta} \frac{1+\cos \left[\frac{2 \mathcal{B}}{\lambda}\left(1-e^{-\lambda \theta}\right)\right]}{2-\mathcal{A}\left\{1-\cos \left[\frac{2 \mathcal{B}}{\lambda}\left(1-e^{-\lambda \theta}\right)\right]\right\}}
$$

Observe that when $\beta_{0}=0, \mathcal{A}=1, \mathcal{F}(\theta)$ in Equation (64) reduces to

$$
\mathcal{F}_{\text {exponential-decay }}^{\text {(on-resonance) }}(\theta)=4 \mathcal{B}^{2} e^{-2 \lambda \theta} .
$$

Lastly, using Equations (37) and (64), the geodesic equation to integrate in this third scenario can be formally written as

$$
\frac{d^{2} \theta}{d \xi^{2}}+\frac{1}{2 \mathcal{F}_{\exp }(\theta)} \frac{d \mathcal{F}_{\exp }(\theta)}{d \theta}\left(\frac{d \theta}{d \xi}\right)^{2}=0,
$$

with $\mathcal{F}_{\exp }(\theta) \stackrel{\text { def }}{=} \mathcal{F}_{\text {exponential-decay }}^{\text {(off-renance }}(\theta)$ as in Equation (64). Making use of a symbolic mathematical software (Mathematica, for instance) together with the analytical knowledge of what happens in the limiting case of $\beta_{0}=0$ [21], Equation (66) can be finally recast as

$$
\frac{d^{2} \theta}{d \xi^{2}}-\left\{\lambda+\frac{(1-\mathcal{A}) \mathcal{B} e^{-\lambda \theta} \tan \left[\frac{\mathcal{B}}{\lambda}\left(1-e^{-\lambda \theta}\right)\right]}{1-\mathcal{A} \sin ^{2}\left[\frac{\mathcal{B}}{\lambda}\left(1-e^{-\lambda \theta}\right)\right]}\right\}\left(\frac{d \theta}{d \xi}\right)^{2}=0 .
$$

In Figure 2, we report the numerical plots of the geodesic paths $\theta$ versus the affine parameter $\xi$ for the four driving strategies with geodesic equations in Equations (46), (53), (60) and (67). In the LHS of Figure 2, we consider the on-resonance scenario with $\beta_{0}=0$. In the RHS of Figure 2, instead, we consider the off-resonance scenario with $\beta_{0}=1 / 2$. In both sides, we set $\Gamma / \hbar=1, \lambda=2 / \pi$, $\theta_{0}=0$, and $\dot{\theta}_{0}=1$. The most noticeable feature in Figure 2 is the deviation from the straight line behavior of the geodesic path that emerges from the constant driving scheme when the on-resonance condition is satisfied. In particular, a first visual comparison of the corresponding plots in the two cases (on-resonance v.s. off-resonance) seems to suggest that the constant driving scheme might be the least robust to departures from the on-resonance condition. Indeed, this preliminary remark will be quantitatively confirmed in our forthcoming information geometric analysis.

\subsection{Geodesic Speeds}

In what follows, after specifying $\Sigma(\theta)$ in Equation (32) and $\mathcal{F}(\theta)$ in Equation (35), we shall focus on finding the geodesic speeds $v$,

$$
v \stackrel{\text { def }}{=} \frac{1}{2} \sqrt{\mathcal{F}(\theta)} \frac{d \theta}{d \xi^{\prime}}
$$

corresponding to the various off-resonant driving schemes. For some technical details on the proof of the fact that geodesic paths have constant speed, we refer to Appendix B.

\subsubsection{Constant Case}

In this first case, using Equations (33), (43) and (44), the on-resonance geodesic speed $v$ from Equation (68) is given by

$$
v_{\mathrm{ON}}=\frac{\Gamma}{\hbar} \dot{\theta}
$$

In the off-resonance regime, instead, the geodesic speed $v$ from Equation (68) can be recast as

$$
v_{\mathrm{OFF}}=\frac{\Gamma}{\hbar}\left\{\frac{1+\cos \left(2 \frac{\Gamma}{\hbar} \sqrt{1+\beta_{0}^{2}} \theta\right)}{2-\frac{1}{1+\beta_{0}^{2}}\left[1-\cos \left(2 \frac{\Gamma}{\hbar} \sqrt{1+\beta_{0}^{2}} \theta\right)\right]}\right\}^{\frac{1}{2}} \dot{\theta} .
$$


Furthermore, setting $\Gamma / \hbar=1, \dot{\theta}\left(\xi_{0}\right)=\dot{\theta}_{0}=1$, and $\theta\left(\xi_{0}\right)=\theta_{0}, v_{\mathrm{ON}}$ and $v_{\mathrm{OFF}}$ in Equations (69) and (70) become

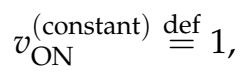

and,

$$
v_{\mathrm{OFF}}^{\text {(constant) }}\left(\beta_{0}, \theta_{0}\right) \stackrel{\text { def }}{=}\left\{\frac{1+\cos \left(2 \sqrt{1+\beta_{0}^{2}} \theta_{0}\right)}{2-\frac{1}{1+\beta_{0}^{2}}\left[1-\cos \left(2 \sqrt{1+\beta_{0}^{2}} \theta_{0}\right)\right]}\right\}^{\frac{1}{2}},
$$

respectively. From Equations (71) and (72), we note that the geodesic speed becomes sensitive to the initial condition $\theta_{0}$ when departing from the on-resonance scenario in the case of a driving strategy with constant complex transverse field intensity. Finally, employing Equations (72) and (71), we define the robustness coefficient as the ratio

$$
r_{\text {constant }}\left(\beta_{0}, \theta_{0}\right) \stackrel{\text { def }}{=} \frac{v_{\mathrm{OFF}}^{\text {(constant) }}\left(\beta_{0}, \theta_{0}\right)}{v_{\mathrm{ON}}^{\text {(constant) }}\left(\theta_{0}\right)},
$$

that is,

$$
r_{\text {constant }}\left(\beta_{0}, \theta_{0}\right)=\left\{\frac{1+\cos \left(2 \sqrt{1+\beta_{0}^{2}} \theta_{0}\right)}{2-\frac{1}{1+\beta_{0}^{2}}\left[1-\cos \left(2 \sqrt{1+\beta_{0}^{2}} \theta_{0}\right)\right]}\right\}^{\frac{1}{2}} .
$$

In Figure 3, we plot the analytical (thin solid) and numerical (filled circle) values of the geodesic speed $v_{\mathrm{OFF}}$ in Equation (70) versus the initial condition $\theta_{0}$ in the case of constant driving. In the plot, we set $\beta_{0}=1 / 2, \Gamma / \hbar=1$, and $\dot{\theta}_{0}=1$. The plot in Figure 3 clearly illustrates the emergence of the sensitivity on the initial condition $\theta_{0}$ of the geodesic speed $v_{\mathrm{OFF}}$ for the constant driving scheme when the on-resonance condition is violated. For the sake of completeness, we point out that despite the fact that this sensitivity is present in the remaining three driving strategies even when the on-resonance condition is fulfilled, off-resonance effects substantially enhance this sensitivity as evident from our information geometric analysis of geodesic speeds presented here.

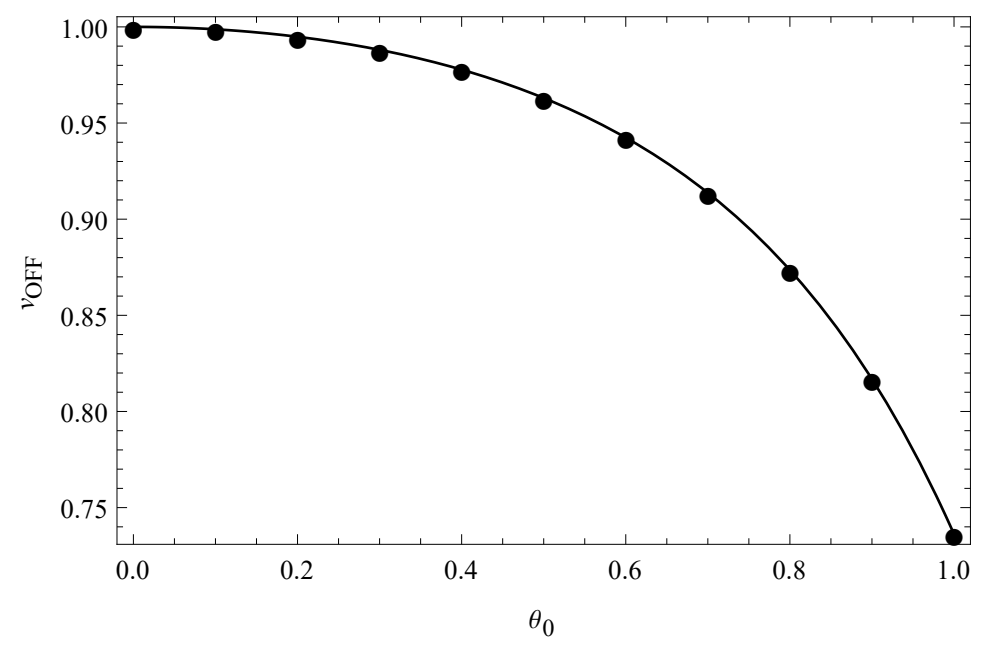

Figure 3. Plot of the analytical (thin solid) and numerical (filled circle) values of the geodesic speed $v_{\mathrm{OFF}}$ versus the initial condition $\theta_{0}$ in the case of constant driving. In the plot, we set $\beta_{0}=1 / 2, \Gamma / \hbar=1$, and $\dot{\theta}_{0}=1$.

\subsubsection{Oscillatory Behavior}

In this second case, making use of Equations (33), (43) and (50), the on-resonance geodesic speed $v$ from Equation (68) becomes

$$
v_{\mathrm{ON}}=\frac{\Gamma}{\hbar}|\cos (\lambda \theta)| \dot{\theta} .
$$


In the off-resonance regime, instead, the geodesic speed $v_{\mathrm{OFF}}$ is given by

$$
v_{\mathrm{OFF}}=\frac{\Gamma}{\hbar}|\cos (\lambda \theta)|\left\{\frac{2 \cos ^{2}\left[\frac{1}{\lambda} \frac{\Gamma}{\hbar} \sqrt{1+\beta_{0}^{2}} \sin (\lambda \theta)\right]}{2-\frac{1}{1+\beta_{0}^{2}}\left\{1-\cos \left[\frac{2}{\lambda} \frac{\Gamma}{\hbar} \sqrt{1+\beta_{0}^{2}} \sin (\lambda \theta)\right]\right\}}\right\}^{\frac{1}{2}} \dot{\theta} .
$$

Moreover, letting $\Gamma / \hbar=1, \lambda=2 / \pi, \dot{\theta}\left(\xi_{0}\right)=\dot{\theta}_{0}=1$, and $\theta\left(\xi_{0}\right)=\theta_{0}, v_{\mathrm{ON}}$ and $v_{\mathrm{OFF}}$ in Equations (75) and (76) become

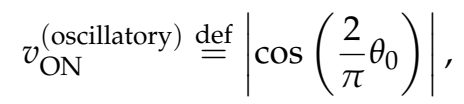

and,

$$
v_{\mathrm{OFF}}^{\text {(oscillatory) }}\left(\beta_{0}, \theta_{0}\right) \stackrel{\text { def }}{=}\left|\cos \left(\frac{2}{\pi} \theta_{0}\right)\right|\left\{\frac{2 \cos ^{2}\left[\frac{\pi}{2} \sqrt{1+\beta_{0}^{2}} \sin \left(\frac{2}{\pi} \theta_{0}\right)\right]}{2-\frac{1}{1+\beta_{0}^{2}}\left\{1-\cos \left[\pi \sqrt{1+\beta_{0}^{2}} \sin \left(\frac{2}{\pi} \theta_{0}\right)\right]\right\}}\right\}^{\frac{1}{2}}
$$

respectively. Finally, using Equations (77) and (78), we introduce the robustness coefficient as the ratio

$$
r_{\text {oscillatory }}\left(\beta_{0}, \theta_{0}\right) \stackrel{\text { def }}{=} \frac{v_{\text {OFF }}^{\text {(oscillatory) }}\left(\beta_{0}, \theta_{0}\right)}{v_{\mathrm{ON}}^{\text {(oscillatory) }}\left(\theta_{0}\right)},
$$

that is,

$$
r_{\text {oscillatory }}\left(\beta_{0}, \theta_{0}\right)=\left\{\frac{2 \cos ^{2}\left[\frac{\pi}{2} \sqrt{1+\beta_{0}^{2}} \sin \left(\frac{2}{\pi} \theta_{0}\right)\right]}{2-\frac{1}{1+\beta_{0}^{2}}\left\{1-\cos \left[\pi \sqrt{1+\beta_{0}^{2}} \sin \left(\frac{2}{\pi} \theta_{0}\right)\right]\right\}}\right\}^{\frac{1}{2}} .
$$

From Equations (77) and (78), we observe that $v_{\mathrm{OFF}}^{\text {(oscillatory) }}\left(\beta_{0}, \theta_{0}\right) \leq v_{\mathrm{ON}}^{\text {(oscillatory) }}$ and $0 \leq$ $r_{\text {oscillatory }}\left(\beta_{0}, \theta_{0}\right)<1$.

\subsubsection{Power Law Decay}

In this third case, Equations (33), (43) and (57) lead to an expression of the on-resonance geodesic speed $v$ from Equation (68) given by

$$
v_{\mathrm{ON}}=\frac{\Gamma}{\hbar} \frac{1}{(1+\lambda \theta)^{2}} \dot{\theta}
$$

In the off-resonance regime where $\beta_{0} \neq 0$, instead, the geodesic speed $v_{\mathrm{OFF}}$ from Equation (68) becomes

$$
v_{\mathrm{OFF}}=\frac{\Gamma}{\hbar} \frac{1}{(1+\lambda \theta)^{2}}\left\{\frac{1+\cos \left(\frac{2 \frac{\Gamma}{\hbar} \sqrt{1+\beta_{0}^{2}} \theta}{1+\lambda \theta}\right)}{2-\frac{1}{1+\beta_{0}^{2}}\left[1-\cos \left(\frac{2 \Gamma \sqrt{1+\beta_{0}^{2}} \theta}{1+\lambda \theta}\right)\right]}\right\}^{\frac{1}{2}} \dot{\theta} .
$$

In addition, assuming $\Gamma / \hbar=1, \lambda=2 / \pi, \dot{\theta}\left(\xi_{0}\right)=\dot{\theta}_{0}=1$, and $\theta\left(\xi_{0}\right)=\theta_{0}, v_{\mathrm{ON}}$ and $v_{\mathrm{OFF}}$ in Equations (81) and (82) become

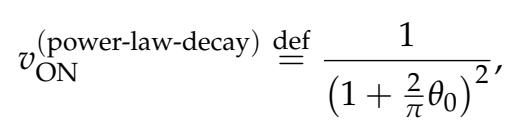


and,

$$
v_{\mathrm{OFF}}^{\text {(power-law-decay) }}\left(\beta_{0}, \theta_{0}\right) \stackrel{\text { def }}{=} \frac{1}{\left(1+\frac{2}{\pi} \theta_{0}\right)^{2}}\left\{\frac{1+\cos \left(\frac{2 \sqrt{1+\beta_{0}^{2}} \theta_{0}}{1+\frac{2}{\pi} \theta_{0}}\right)}{2-\frac{1}{1+\beta_{0}^{2}}\left[1-\cos \left(\frac{2 \sqrt{1+\beta_{0}^{2}} \theta_{0}}{1+\frac{2}{\pi} \theta_{0}}\right)\right]}\right\}^{\frac{1}{2}},
$$

respectively. Lastly, employing Equations (83) and (84), we define the robustness coefficient as the ratio

$$
r_{\text {power-law-decay }}\left(\beta_{0}, \theta_{0}\right) \stackrel{\text { def }}{=} \frac{v_{\text {OFF }}^{\text {(power-law-decay })}\left(\beta_{0}, \theta_{0}\right)}{v_{\text {ON }}^{(\text {power-law-decay })}\left(\theta_{0}\right)},
$$

that is,

$$
r_{\text {power-law-decay }}\left(\beta_{0}, \theta_{0}\right)=\left\{\frac{1+\cos \left(\frac{2 \sqrt{1+\beta_{0}^{2}} \theta_{0}}{1+\frac{2}{\pi} \theta_{0}}\right)}{2-\frac{1}{1+\beta_{0}^{2}}\left[1-\cos \left(\frac{2 \sqrt{1+\beta_{0}^{2}} \theta_{0}}{1+\frac{2}{\pi} \theta_{0}}\right)\right]}\right\}^{\frac{1}{2}} .
$$

From Equations (83) and (84), we observe that $v_{\mathrm{OFF}}^{\text {(power-law-decay) }}\left(\beta_{0}, \theta_{0}\right) \leq v_{\mathrm{ON}}^{\text {(power-law-decay) }}$ and $0 \leq r_{\text {power-law-decay }}\left(\beta_{0}, \theta_{0}\right)<1$.

\subsubsection{Exponential Decay}

In this fourth and last case, making use of Equations (33), (43) and (64), the on-resonance geodesic speed $v$ from Equation (68) becomes

$$
v_{\mathrm{ON}}=\frac{\Gamma}{\hbar} e^{-\lambda \theta} \dot{\theta}
$$

In the off-resonance regime, instead, the geodesic speed $v_{\mathrm{OFF}}$ is given by

$$
v_{\mathrm{OFF}}=\frac{\Gamma}{\hbar} e^{-\lambda \theta}\left\{\frac{1+\cos \left[\frac{2}{\lambda} \frac{\Gamma}{\hbar} \sqrt{1+\beta_{0}^{2}}\left(1-e^{-\lambda \theta}\right)\right]}{2-\frac{1}{1+\beta_{0}^{2}}\left\{1-\cos \left[\frac{2}{\lambda} \frac{\Gamma}{\hbar} \sqrt{1+\beta_{0}^{2}}\left(1-e^{-\lambda \theta}\right)\right]\right\}}\right\}^{\frac{1}{2}} \dot{\theta} .
$$

Assuming $\Gamma / \hbar=1, \lambda=2 / \hbar, \dot{\theta}\left(\xi_{0}\right)=\dot{\theta}_{0}=1$, and $\theta\left(\xi_{0}\right)=\theta_{0}, v_{\mathrm{ON}}$ and $v_{\mathrm{OFF}}$ in Equations (87) and (88) become

$$
v_{\text {ON }}^{(\text {exponential-decay })} \stackrel{\text { def }}{=} e^{-\frac{2}{\pi} \theta_{0}},
$$

and,

$$
v_{\mathrm{OFF}}^{(\text {exponential-decay })}\left(\beta_{0}, \theta_{0}\right) \stackrel{\text { def }}{=} e^{-\frac{2}{\pi} \theta_{0}}\left\{\frac{1+\cos \left[\pi \sqrt{1+\beta_{0}^{2}}\left(1-e^{-\frac{2}{\pi} \theta_{0}}\right)\right]}{2-\frac{1}{1+\beta_{0}^{2}}\left\{1-\cos \left[\pi \sqrt{1+\beta_{0}^{2}}\left(1-e^{-\frac{2}{\pi} \theta_{0}}\right)\right]\right\}}\right\}^{\frac{1}{2}},
$$

respectively. Finally, by means of Equations (89) and (90), we define the robustness coefficient as the ratio

$$
r_{\text {exponential-decay }}\left(\beta_{0}, \theta_{0}\right) \stackrel{\text { def }}{=} \frac{v_{\text {OFF }}^{\text {(exponential-decay) }}\left(\beta_{0}, \theta_{0}\right)}{v_{\mathrm{ON}}^{\text {(exponential-decay) }}\left(\theta_{0}\right)},
$$

that is,

$$
r_{\text {exponential-decay }}\left(\beta_{0}, \theta_{0}\right)=\left\{\frac{1+\cos \left[\pi \sqrt{1+\beta_{0}^{2}}\left(1-e^{-\frac{2}{\pi} \theta_{0}}\right)\right]}{2-\frac{1}{1+\beta_{0}^{2}}\left\{1-\cos \left[\pi \sqrt{1+\beta_{0}^{2}}\left(1-e^{-\frac{2}{\pi} \theta_{0}}\right)\right]\right\}}\right\}^{\frac{1}{2}} .
$$


In Figure 4, going from left to right, we plot: (L) Plot of the geodesic speeds $v_{\mathrm{ON}}$ in Equations (69), (75), (81) and (87) versus the initial condition $\theta_{0}$ with $\beta_{0}=0$; (C) Plot of the geodesic speeds $v_{\mathrm{OFF}}$ in Equations (70), (76), (82) and (88) versus the initial condition $\theta_{0}$ with $\beta_{0}=1 / 2$; (R) Plot of the robustness coefficients $r$ in Equations (73), (79), (85) and (91) versus the affine parameter $\xi$. Plots that correspond to the constant, oscillatory, exponential decay, and power law decay driving strategies appear in dotted, dashed, thin solid, and thick solid lines, respectively. In all plots, we set $\Gamma / \hbar=1, \lambda=2 / \pi$, and $\dot{\theta}_{0}=1$. The plots in Figure 4 help exhibiting the effects of the off-resonance condition on the speed of geodesic paths that correspond to the variety of driving strategies being considered in this paper. In particular, we observe that to faster (slower) driving strategies, there seem to correspond smaller (larger) robustness coefficients. In other words, slower driving strategies appear to be more robust against departures from the on-resonance condition. The ranking of the various driving strategies is quite straightforward in the on-resonance condition. However, when departing from this condition, off-resonance effects lead to a richer set of dynamical scenarios. This, in turn, makes the comparison of the performance of our chosen driving schemes more delicate. For example, despite our illustrative depiction in Figure 4, it is possible to uncover two-dimensional parametric regions $\mathcal{P}\left(\beta_{0}, \theta_{0}\right)$ specified by the parameters $\beta_{0}$ and $\theta_{0}$ where the constant driving scheme is not only the least robust but also the slowest one. Indeed, we plot in Figure 5 a two-dimensional parametric region $\mathcal{P}\left(\beta_{0}, \theta_{0}\right)$,

$$
\mathcal{P}\left(\beta_{0}, \theta_{0}\right) \stackrel{\text { def }}{=}\left\{\left(\beta_{0}, \theta_{0}\right) \in \mathbb{R}_{+} \times \mathbb{R}_{+}: v_{\mathrm{OFF}}^{(\text {strategy }-i)} \geq v_{\mathrm{OFF}}^{(\text {strategy }-j)} \text { and, } r^{(\text {strategy }-i)} \geq r^{(\text {strategy }-j)}\right\},
$$

with $i \neq j \in\{1,2,3,4\}$ and where the oscillatory (light grey), the exponential decay (grey), and the power law decay (black) driving strategies outperform the constant driving scheme in terms of both geodesic speed and robustness. More specifically, in Figure 5 we have

$$
\mathcal{P}_{\text {power-law-decay }}\left(\beta_{0}, \theta_{0}\right) \subseteq \mathcal{P}_{\text {exponential-decay }}\left(\beta_{0}, \theta_{0}\right) \subseteq \mathcal{P}_{\text {oscillatory }}\left(\beta_{0}, \theta_{0}\right) ，
$$

and, in addition,

$$
r_{\text {power-law-decay }}\left(\beta_{0}, \theta_{0}\right) \geq r_{\text {exponential-decay }}\left(\beta_{0}, \theta_{0}\right) \geq r_{\text {oscillatory }}\left(\beta_{0}, \theta_{0}\right) \text {. }
$$
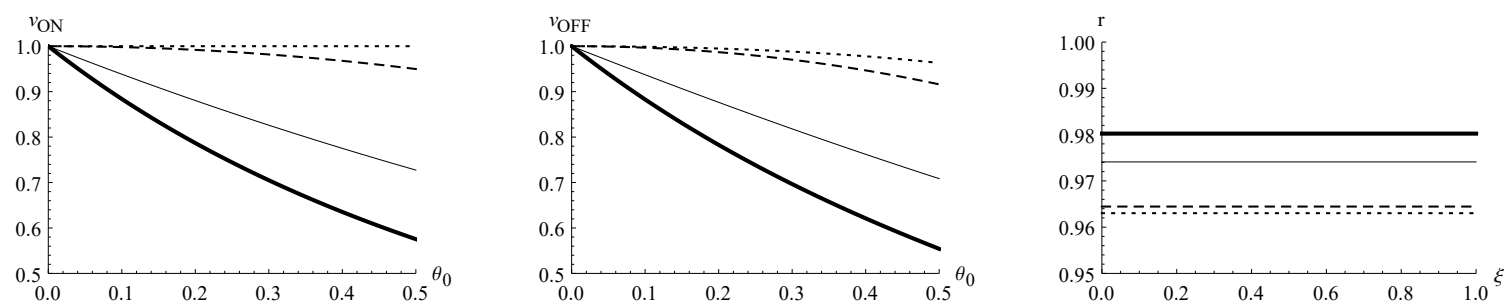

Figure 4. From left to right: (L) Plot of the geodesic speed $v_{\mathrm{ON}}$ versus the initial condition $\theta_{0}$ with $\beta_{0}=0 ;(\mathbf{C})$ Plot of the geodesic speed $v_{\mathrm{OFF}}$ versus the initial condition $\theta_{0}$ with $\beta_{0}=1 / 2$; (R) Plot of the robustness coefficient $r$ versus the affine parameter $\xi$. Plots that correspond to the constant, oscillatory, exponential decay, and power law decay strategies appear in dotted, dashed, thin solid, and thick solid lines, respectively. In all plots, we set $\Gamma / \hbar=1, \lambda=2 / \pi$, and $\dot{\theta}_{0}=1$.

In all region plots, we set $\Gamma / \hbar=1, \lambda=2 / \pi$, and $\dot{\theta}_{0}=1$. In addition, the maximum success probability of each driving scheme is assumed to be greater than $25 / 26$, that is, $0 \leq \beta_{0} \lesssim 0.20$ in Figure 5 . The main take-home message from Figure 5 is that in the off-resonance scenario it is possible to uncover two-dimensional parametric regions $\mathcal{P}\left(\beta_{0}, \theta_{0}\right)$ where the best on-resonance driving scheme (that is, the constant one) can be outperformed both in terms of speed and robustness. 


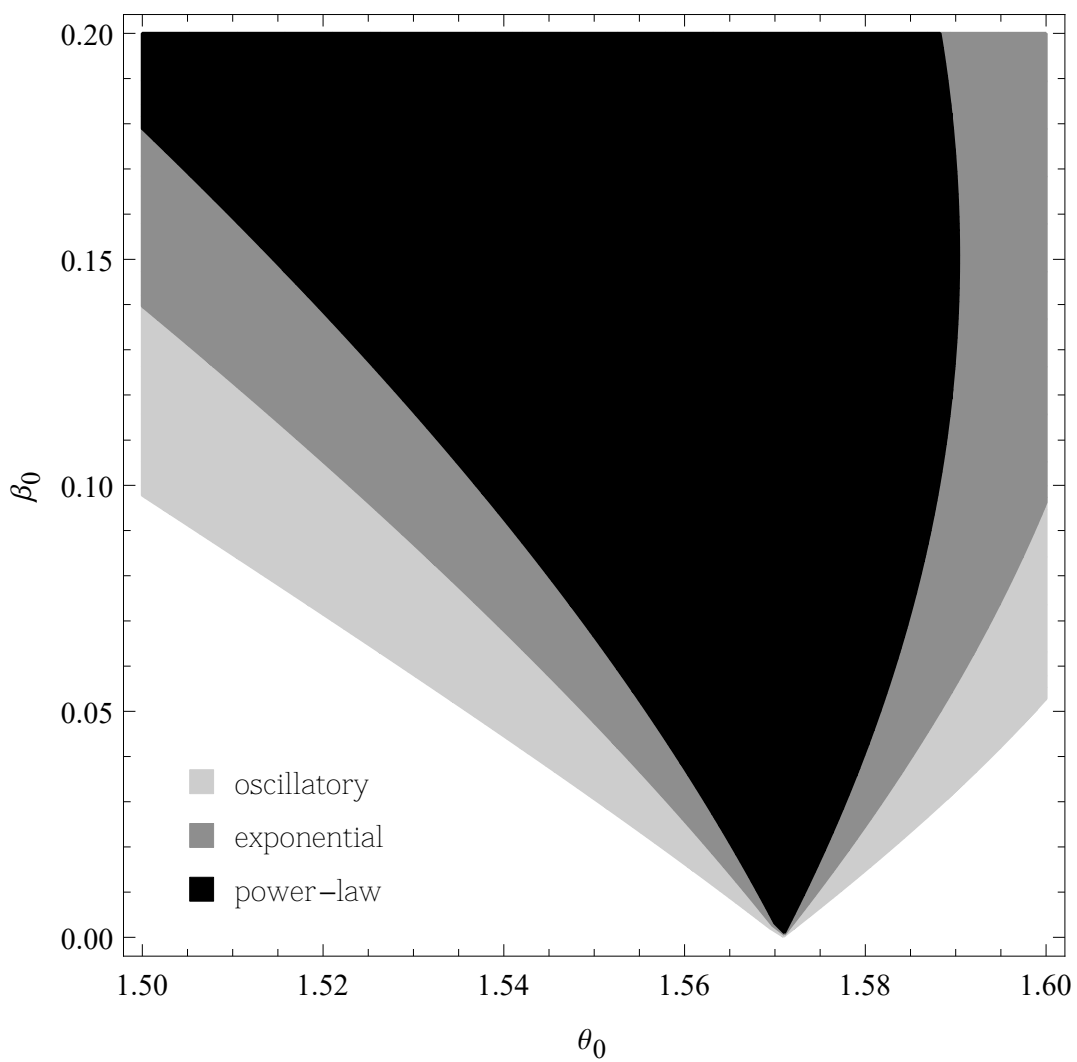

Figure 5. Plot of the two-dimensional parametric regions $\mathcal{P}\left(\beta_{0}, \theta_{0}\right)$ where the oscillatory (light grey), the exponential decay (grey), and the power law decay (black) driving strategies outperform the constant driving scheme in terms of both geodesic speed and robustness. Note that the light grey region contains the grey region while the grey region contains the black region. Furthermore, the black region specifies a driving strategy more robust than that specified by the grey region while the grey region specifies a driving strategy more robust than that specified by the light grey region. In all region plots, we set $\Gamma / \hbar=1, \lambda=2 / \pi$, and $\dot{\theta}_{0}=1$. Finally, the maximum success probability of each driving scheme is assumed to be greater than $25 / 26 \simeq 0.96$, that is, $0 \leq \beta_{0} \lesssim 0.20$.

\section{Conclusions}

In this paper, we presented an information geometric analysis of off-resonance effects on classes of exactly solvable generalized semi-classical Rabi systems. Specifically, we considered population transfer performed by four distinct off-resonant driving schemes specified by su $(2 ; \mathbb{C})$ time-dependent Hamiltonian models. For each scheme, we studied the consequences of a departure from the on-resonance condition in terms of both geodesic paths and geodesic speeds on the corresponding manifold of transition probability vectors. In particular, we analyzed the robustness of each driving scheme against off-resonance effects. Moreover, we reported on a possible tradeoff between speed and robustness in the driving schemes being investigated. Finally, we discussed the emergence of a different relative ranking in terms of performance among the various driving schemes when transitioning from the on-resonant to the off-resonant scenarios.

Our main findings can be outlined as follows.

(1) In the presence of off-resonance effects, the success probability of the various quantum strategies is affected both in terms of amplitude and periodicity. In particular, focusing on the constant driving case, the success probability $p_{0}(\theta)$ in Equation (42) is dampened by the Lorentzian-like factor $1 /\left(1+\beta_{0}^{2}\right)$. Moreover, the periodicity of the oscillations of this probability changes from $2 \pi$ to $2 \pi /\left(1+\beta_{0}^{2}\right)^{\frac{1}{2}}$. In summary, oscillations become smaller in amplitude but higher in frequency. These facts are clearly visible in Figure 1. 
(2) Departing from the on-resonance condition (that is, $\beta_{0}=0$ ), we observe a change in the geodesic paths on the underlying manifolds. The presence of off-resonance effects (that is, $\beta_{0} \neq 0$ ) generates geodesic paths with a more complex structure. In particular, unlike what happens when the on-resonance condition is satisfied, numerical integration of the geodesic equations is required. In particular, the numerical plots of the geodesic paths $\theta$ versus the affine parameter $\xi$ for the four driving strategies with geodesic equations in Equations (46), (53), (60) and (67) appear in Figure 2.

(3) Each and every numerical value of the geodesic speeds corresponding to the various quantum driving strategies considered in this paper becomes smaller when departing from the on-resonance condition (see Figure 3, for instance). In general, quantum driving strategies characterized by a high geodesic speed appear to be very sensitive to off-resonance effects. Instead, strategies yielding geodesic paths with smaller geodesic speed values seem to be more robust against departures from the on-resonance condition. These observations can be understood from Figure 4.

(4) In the off-resonance regime, there emerges a sensitive dependence of the geodesic speeds on the initial conditions. This, in turn, can cause a change in the ranking of the various quantum driving strategies. In particular, the strategy specified by a constant Fisher information is no longer the absolute best strategy in terms of speed. Indeed, it is possible to find two-dimensional (2D) parametric regions where this strategy is being outperformed by all the remaining strategies both in terms of speed and robustness. In general, these 2D regions are more extended for the less robust strategies (see Equations (94) and (95)). For instance, we can identify 2D regions where the speed-based ranking becomes: (1) Oscillatory strategy; (2) exponential-law decay strategy; (3) power-law decay strategy. Instead, considering the very same 2D regions, the robustness-based ranking is given by: (1) Power-law decay strategy; (2) exponential-law decay strategy; (3) oscillatory strategy. In these 2D regions, the constant strategy is the worst, both in terms of speed and robustness. These findings are illustrated in Figure 5.

In conclusion, our information geometric analysis suggests that speed and robustness appear to be competing features when departing from the on-resonance condition. Ideally, a quantum driving strategy should be fast, robust, and thermodynamically efficient [34-36]. Since speed and thermodynamic efficiency quantified in terms of minimum entropy production paths seem to be conflicting properties as well $[20,21]$, it appears reasonable to think there might be a connection between robustness and thermodynamic efficiency. We leave the exploration of this conjectured link to future scientific efforts.

Author Contributions: Conceptualization, C.C.; Numeri- cal computations, S.G. and C.C.; Writing-original draft, C.C.; Writing-review and editing, C.C., S.G., and P.M.A.; Supervision, P.M.A. All authors have read and agreed to the published version of the manuscript.

Funding: C.C. acknowledges support under AFRL Visiting Faculty Fellowship Program, AFRL Contract No. FA8750-15-3-6003.

Acknowledgments: C.C. is grateful to the United States Air Force Research Laboratory (AFRL) Summer Faculty Fellowship Program for providing support for this work. Any opinions, findings and conclusions or recommendations expressed in this manuscript are those of the authors and do not necessarily reflect the views of AFRL.

Conflicts of Interest: The authors declare no conflict of interest.

\section{Appendix A. Violating a Resonance Condition}

In this Appendix, we provide two illustrative examples concerning the violation of a resonance condition. 
Appendix A.1. A Classical Scenario

In the framework of classical mechanics, one can imagine violating a resonance condition in a number of manners. For instance, consider a mass-spring system in the presence of damping and sinusoidal forcing term,

$$
m \ddot{x}+\lambda \dot{x}+k x=f_{0} \cos (\gamma t) .
$$

In Equation (A1), $\dot{x} \stackrel{\text { def }}{=} d x / d t$. Furthermore, $m, \lambda, k$, and $f_{0}$ denote the mass, the damping coefficient, the spring constant, and the amplitude of the forcing term, respectively. The classical resonance curve $\mathcal{C}(\gamma)$,

$$
\mathcal{C}(\gamma) \stackrel{\text { def }}{=}\left[\left(k-m \gamma^{2}\right)^{2}+b^{2} \gamma^{2}\right]^{-1 / 2}
$$

for this specific physical system is proportional to the amplitude $\mathcal{A}(\gamma) \stackrel{\text { def }}{=} f_{0} \mathcal{C}(\gamma)$ of the steady-state solution to Equation (A1). Moreover, the resonance condition $\gamma=\gamma^{*}$ with

$$
\gamma^{*}=\gamma^{*}(m, k, \lambda) \stackrel{\text { def }}{=}\left(\frac{k}{m}-\frac{\lambda^{2}}{2 m^{2}}\right)^{1 / 2}
$$

is specified by imposing the maximum $\overline{\mathcal{C}} \stackrel{\text { def }}{=} \mathcal{C}\left(\gamma^{*}\right)$ of the resonance curve $\mathcal{C}(\gamma)$ in Equation (A2). Therefore, we clearly note from Equation (A3) that departures from the resonance condition $\gamma=\gamma^{*}$ can happen by varying the stiffness $(k)$, the mass $(m)$, and/or the damping $(\lambda)$.

\section{Appendix A.2. A Quantum Scenario}

In the framework of quantum mechanics, one can imagine violating a resonance condition in a number of manners. For instance, consider a two-level quantum system described by a time-dependent Hamiltonian,

$$
\mathcal{H}(t) \stackrel{\text { def }}{=} \mathcal{H}_{0}+\mathcal{V}(t),
$$

where $\mathcal{H}_{0}$ is the part of the Hamiltonian $\mathcal{H}$ that does not contain time explicitly while $\mathcal{V}(t)$ is the time-dependent sinusoidal oscillatory potential. The quantities $\mathcal{H}_{0}$ and $\mathcal{V}(t)$ are defined as,

$$
\mathcal{H}_{0} \stackrel{\text { def }}{=} E_{1}|1\rangle\left\langle 1\left|+E_{2}\right| 2\right\rangle\left\langle 2\left|, \mathcal{V}(t) \stackrel{\text { def }}{=} \gamma e^{i \omega t}\right| 1\right\rangle\left\langle 2\left|+\gamma e^{-i \omega t}\right| 2\right\rangle\langle 1|,
$$

respectively, where $\gamma$ and $\omega$ belong to $\mathbb{R}_{+} \backslash\{0\}$. Furthermore, $|1\rangle$ and $|2\rangle$ are two eigenstates of $\mathcal{H}_{0}$ with corresponding eigenvalues $E_{1}$ and $E_{2}$, respectively, with $E_{2}>E_{1}$. The quantum resonance curve $\mathcal{Q}(\omega)$

$$
\mathcal{Q}(\omega) \stackrel{\text { def }}{=}\left[1+\left(\frac{\hbar}{\gamma}\right)^{2} \frac{\left(\omega-\omega_{21}\right)^{2}}{4}\right]^{-1},
$$

for this specific physical system described by the Hamiltonian in Equation (A4) is the amplitude squared that characterizes the transition probability $\mathcal{P}_{|1\rangle \rightarrow|2\rangle}$ (assuming that at $t=0$ only the level $|1\rangle$ is populated) between the two quantum states $|1\rangle$ and $|2\rangle$ with $\omega_{21} \stackrel{\text { def }}{=}\left(E_{2}-E_{1}\right) / \hbar$ being the characteristic frequency of the system. Moreover, the resonance condition $\omega=\omega^{*}$ with

$$
\omega^{*}=\omega_{21}=\omega_{21}\left(E_{1}, E_{2}\right) \stackrel{\text { def }}{=}\left(E_{2}-E_{1}\right) / \hbar,
$$

is specified by imposing the maximum $\overline{\mathcal{Q}} \stackrel{\text { def }}{=} \mathcal{Q}\left(\omega_{21}\right)$ of the resonance curve $\mathcal{Q}(\omega)$ in Equation (A6). Therefore, we clearly note from Equation (A7) that departures from the resonance condition $\omega=\omega^{*}$ 
can happen by varying the larger $\left(E_{2}\right)$ and the smaller $\left(E_{1}\right)$ energy levels. We point out that considering the following correspondences

$$
\omega \rightarrow \omega, \gamma \rightarrow \frac{|e| \hbar B_{\perp}}{2 m c} \text {, and } \omega_{21} \rightarrow \frac{|e| B_{\|}}{m c},
$$

it can be shown that the Hamiltonian in Equation (A4) describes a two-level quantum mechanical system represented by a spin-1/2 particle immersed in an external magnetic field $\vec{B}(t)$ given by,

$$
\vec{B}(t) \stackrel{\text { def }}{=} B_{\|} \hat{z}+B_{\perp}[\cos (\omega t) \hat{x}+\sin (\omega t) \hat{y}] .
$$

The resonance condition $\omega=\omega^{*}$ is satisfied whenever the positively assumed frequency $\omega$ of the rotating magnetic field in the $x y$-plane equals Larmor's precessional frequency specified by the intensity of the uniform magnetic field $B_{\|} \hat{z}[2]$,

$$
\omega^{*}=\omega^{*}\left(|e|, B_{\|}, m, c\right) \stackrel{\text { def }}{=} \frac{|e| B_{\|}}{m c} .
$$

Finally, we emphasize that the analogue of the adimensional parameter $\beta_{0}$ used throughout our paper becomes in this quantum mechanical framework the quantity defined as

$$
\beta_{0} \stackrel{\text { def }}{=} \frac{m c}{|e| B_{\perp}}\left(\omega-\omega^{*}\right)=\frac{m c}{|e| B_{\perp}}\left(\omega-\frac{|e| B_{\|}}{m c}\right) .
$$

Observe that Equation (A11) is the static version of Equation (23). For further details on the quantum mechanical motion in two-level quantum systems described by time-dependent Hamiltonians, we refer to reference [2].

\section{Appendix B. Speed of Geodesics}

In the Appendix, we briefly show that geodesics have constant speed. For further details, we refer to reference [37].

Recall that if $\Theta(\xi) \stackrel{\text { def }}{=}\left(\theta^{1}(\xi), \ldots, \theta^{n}(\xi)\right)$ is a curve in a $n$-dimensional Riemannian manifold $\mathcal{M}$, the speed of $\Theta$ at any time is the length of its velocity vector $\|\dot{\Theta}(\xi)\|$,

$$
\|\dot{\Theta}(\xi)\| \stackrel{\text { def }}{=}\langle\dot{\Theta}(\xi), \dot{\Theta}(\xi)\rangle^{\frac{1}{2}}=\left[g_{a b}(\theta) \dot{\theta}^{a} \dot{\theta}^{b}\right]^{\frac{1}{2}},
$$

where $g_{a b}(\theta)$ denotes a Riemannian metric on the manifold $\mathcal{M}$ while $\left\{\theta^{a}(\xi)\right\}$ are the component functions of the curve $\Theta(\xi)$. We say $\Theta(\xi)$ is constant speed if $\|\dot{\Theta}(\xi)\|$ in Equation (A12) does not depend on $\xi$, and unit speed if the speed is identically equal to one.

It is well-known that all Riemannian geodesics are constant speed curves. Indeed, let $\mathcal{M}$ be a manifold with a linear connection $\nabla$, and let $\Theta$ be a curve on $\mathcal{M}$. The acceleration of $\Theta$ is the vector field $D_{\xi} \dot{\Theta}$ along $\Theta$ (that is, the covariant derivative of $\dot{\Theta}(\xi)$ along $\Theta$ ). A curve $\Theta$ is called a geodesic with respect to $\nabla$ if its acceleration $D_{\tilde{\xi}} \dot{\Theta}$ equals zero (that is, the velocity vector field $\dot{\Theta}$ is parallel along the curve $\Theta$ ). Therefore, for any geodesic $\Theta(\xi)$, we have

$$
\begin{aligned}
\frac{d}{d \xi}\left[\|\dot{\Theta}(\xi)\|^{2}\right] & =\frac{d}{d \xi}[\langle\dot{\Theta}(\xi), \dot{\Theta}(\xi)\rangle] \\
& =\left\langle D_{\xi} \dot{\Theta}(\xi), \dot{\Theta}(\xi)\right\rangle+\left\langle\dot{\Theta}(\xi), D_{\tilde{\xi}} \dot{\Theta}(\xi)\right\rangle \\
& =0,
\end{aligned}
$$


that is,

$$
\frac{d}{d \xi}\left[\|\dot{\Theta}(\xi)\|^{2}\right]=0 .
$$

From Equation (A14), we conclude that geodesics $\{\Theta(\xi)\}$ have constant speed $\{\|\dot{\Theta}(\xi)\|\}$.

\section{References}

1. Jackson, J.D. Classical Electrodynamics; John Wiley \& Sons, Inc.: New York, NY, USA, 1999.

2. Sakurai, J.J. Modern Quantum Mechanics; Addison-Wesley Publishing Company, Inc.: Boston, MA, USA, 1994.

3. D'Alessandro, D.; Dahleh, M. Optimal control of two-level quantum systems. IEEE Trans. Autom. Control 2001, 46, 866. [CrossRef]

4. Boscain, U.; Charlot, G.; Gauthier, J.-P.; Guerin, S.; Jauslin, H.-R. Optimal control in laser-induced population transfer for two- and three-level quantum systems. J. Math. Phys. 2002, 43, 2107. [CrossRef]

5. Romano, R. Geometric analysis of minimum-time trajectories for a two-level quantum system. Phys. Rev. A 2014, 90, 062302. [CrossRef]

6. Byrnes, T.; Forster, G.; Tessler, L. Generalized Grover's algorithm for multiple phase inversion states. Phys. Rev. Lett. 2018, 120, 060501. [CrossRef]

7. Cafaro, C.; Alsing, P.M. Continuous-time quantum search and time-dependent two-level quantum systems. Int. J. Quantum Inf. 2019, 17, 1950025. [CrossRef]

8. Aharonov, Y.; Anandan, J.; Popescu, S.; Vaidman, L. Superpositions of time evolutions of a quantum system and a quantum time-translation machine. Phys. Rev. Lett. 1990, 64, 2965. [CrossRef]

9. Kempf, A.; Prain, A. Driving quantum systems with Superoscillations. J. Math. Phys. 2017, 58, 082101. [CrossRef]

10. Barredo, D.; Labuhn, H.; Ravets, S.; Lahaye, T.; Browaeys, A. Coherent excitation transfer in a spin chain of three Rydberg atoms. Phys. Rev. Lett. 2015, 114, 113002. [CrossRef]

11. Schempp, H.; Gunter, G.; Wuster, S.; Weidemuller, M.; Whitlock, S. Correlated exciton transport in Rydberg-dressed-atom spin chains. Phys. Rev. Lett. 2015, 115, 093002. [CrossRef]

12. Shi, Z.C.; Wang, W.; Yi, X.X. Population transfer driven by off-resonant fields. Opt. Express 2016, 24, 21971. [CrossRef]

13. Hirose, M.; Cappellaro, P. Time-optimal control with finite bandwidth. Quantum Inf. Process. 2018, 17, 88. [CrossRef]

14. Allen, L.; Eberly, J.H. Optical Resonance and Two-Level Atoms; Dover Publications: New York, NY, USA, 1987.

15. Laucht, A.; Simmons, S.; Kalra, R.; Tosi, G.; Dehollain, J.P.; Muhonen, J.T.; Freer, S.; Hudson, F.E.; Itoh, K.M.; Jamieson, D.N.; et al. Breaking the rotating wave approximation for a strongly driven dressed single-electron spin. Phys. Rev. B 2016, 94, 161302(R). [CrossRef]

16. Dai, K.; Wu, H.; Zhao, P.; Li, M.; Liu, Q.; Xue, G.; Tan, X.; Yu, H.; Yu, Y. Quantum simulation of the general semi-classical Rabi model in regimes of arbitrarily strong driving. Appl. Phys. Lett. 2017, 111, 242601. [CrossRef]

17. Cafaro, C.; Alsing, P.M. Theoretical analysis of a nearly optimal analog quantum search. Phys. Scr. 2019, 94, 085103. [CrossRef]

18. Gassner, S.; Cafaro, C.; Capozziello, S. Transition probabilities in generalized quantum search Hamiltonian evolutions. Int. J. Geom. Methods Mod. Phys. 2020, accepted. [CrossRef]

19. Farhi, E.; Gutmann, S. Analog analogue of a digital quantum computation. Phys. Rev. A 1998, 57, 2403. [CrossRef]

20. Cafaro, C.; Alsing, P.M. Decrease of Fisher information and the information geometry of evolution equations for quantum mechanical probability amplitudes. Phys. Rev. E 2018, 97, 042110. [CrossRef] [PubMed]

21. Cafaro, C.; Alsing, P.M. Information geometry aspects of minimum entropy production paths from quantum mechanical evolutions. Phys. Rev. E 2019, in press.

22. Messina, A.; Nakazato, H. Analytically solvable Hamiltonians for quantum two-level systems and their dynamics. J. Phys. A Math. Theor. 2014, 47, 445302. [CrossRef]

23. Grimaudo, R.; de Castro, A.S.M.; Nakazato, H.; Messina, A. Classes of exactly solvable generalized semi-classical Rabi systems. Ann. Phys. 2018, 2018, 1800198. [CrossRef] 
24. Braunstein, S.L.; Caves, C.M. Statistical distance and geometry of quantum states. Phys. Rev. Lett. 1994, 72, 3439. [CrossRef] [PubMed]

25. Berry, M.V. Quantal phase factors accompanying adiabatic changes. Proc. R. Soc. Lond. A 1984, $392,45$.

26. Aharonov, Y.; Anandan, J. Phase change during a cyclic quantum evolution. Phys. Rev. Lett. 1987, $58,1593$. [CrossRef] [PubMed]

27. Resta, R. Manifestations of Berry's phase in molecules and condensed matter. J. Phys. Condens. Matter 2000, 12, R107. [CrossRef]

28. Braunstein, S.L.; Caves, C.M.; Milburn, G.J. Generalized uncertainty relations: Theory, examples, and Lorenz Invariance. Ann. Phys. 1996, 247, 135. [CrossRef]

29. Cafaro, C.; Mancini, S. An information geometric viewpoint of algorithms in quantum computing. AIP Conf. Proc. 2012, 1443, 374.

30. Cafaro, C.; Mancini, S. On Grover's search algorithm from a quantum information geometry viewpoint. Physica A 2012, 391, 1610. [CrossRef]

31. Cafaro, C. Geometric algebra and information geometry for quantum computational software. Physica $A$ 2017, 470, 154. [CrossRef]

32. Amari, S.; Nagaoka, H. Methods of Information Geometry; Oxford University Press: Providence, RI, USA, 2000.

33. Felice, D.; Cafaro, C.; Mancini, S. Information geometric methods for complexity. Chaos 2018, 28, 032101. [CrossRef]

34. Castelvecchi, D. Clash of the physical laws. Nature 2017, 543, 597. [CrossRef]

35. Faist, P.; Renner, R. Fundamental work cost of quantum processes. Phys. Rev. X 2018, 8, 021011. [CrossRef]

36. Campbell, S.; Deffner, S. Trade-off between speed and cost in shortcuts to adiabaticity. Phys. Rev. Lett. 2017, 118, 100601. [CrossRef] [PubMed]

37. Lee, J.M. Riemannian Manifolds: An Introduction to Curvature; Springer: New York, NY, USA, 1997.

(C) 2020 by the authors. Licensee MDPI, Basel, Switzerland. This article is an open access article distributed under the terms and conditions of the Creative Commons Attribution (CC BY) license (http:/ / creativecommons.org/licenses/by/4.0/). 